ANALYSIS \& PDE

Volume $2 \quad$ No. $3 \quad 2009$

\author{
TADAHIRO OH
}

PERIODIC STOCHASTIC KORTEWEG-DE VRIES EQUATION

WITH ADDITIVE SPACE-TIME WHITE NOISE 


\title{
PERIODIC STOCHASTIC KORTEWEG-DE VRIES EQUATION WITH ADDITIVE SPACE-TIME WHITE NOISE
}

\author{
TADAHIRO OH
}

\begin{abstract}
We prove the local well-posedness of the periodic stochastic Korteweg-de Vries equation with the additive space-time white noise. To treat low regularity of the white noise in space, we consider the Cauchy problem in the Besov-type space $\hat{b}_{p, \infty}^{s}(\mathbb{T})$ for $s=-\frac{1}{2}+, p=2+$ such that $s p<-1$. In establishing local well-posedness, we use a variant of the Bourgain space adapted to $\hat{b}_{p, \infty}^{s}(\mathbb{T})$ and establish a nonlinear estimate on the second iteration on the integral formulation. The deterministic part of the nonlinear estimate also yields the local well-posedness of the deterministic $\mathrm{KdV}$ in $M(\mathbb{T})$, the space of finite Borel measures on $\mathbb{T}$.
\end{abstract}

\section{Introduction}

In this paper, we prove the local well-posedness of the periodic stochastic Korteweg-de Vries (SKdV) equation with additive space-time white noise:

$$
\left\{\begin{array}{l}
d u+\left(\partial_{x}^{3} u+u \partial_{x} u\right) d t=d W \\
u(x, 0)=u_{0}(x)
\end{array}\right.
$$

where $u$ is a real-valued function, $(x, t) \in \mathbb{T} \times \mathbb{R}^{+}$with $\mathbb{T}=[0,2 \pi)$, and $W(t)=\partial B / \partial x$ is a cylindrical Wiener process on $L^{2}(\mathbb{T})$. With $e_{n}(x)=\frac{1}{\sqrt{2 \pi}} e^{i n x}$, we have $W(t)=\beta_{0}(t) e_{0}+\sum_{n \neq 0} \frac{1}{\sqrt{2}} \beta_{n}(t) e_{n}(x)$, where $\left\{\beta_{n}\right\}_{n \geq 0}$ is a family of mutually independent complex-valued Brownian motions (here we take $\beta_{0}$ to be real-valued) in a fixed probability space $(\Omega, \mathscr{F}, P)$ associated with a filtration $\left\{\mathscr{F}_{t}\right\}_{t \geq 0}$ and $\beta_{-n}(t)=\overline{\beta_{n}}(t)$ for $n \geq 1$. Note that $\operatorname{Var}\left(\beta_{n}(1)\right)=2$ for $n \geq 1$.

De Bouard et al. [2004] considered

$$
\left\{\begin{array}{l}
d u+\left(\partial_{x}^{3} u+u \partial_{x} u\right) d t=\phi d W, \\
u(x, 0)=u_{0}(x),
\end{array}\right.
$$

where $\phi$ is a bounded linear operator in $L^{2}(\mathbb{T})$. They showed that (2) is locally well posed when $\phi$ is a Hilbert-Schmidt operator from $L^{2}(\mathbb{T})$ to $H^{s}(\mathbb{\mathbb { T }})$ for $s>-\frac{1}{2}$. See the references in their paper for earlier work in the periodic and nonperiodic settings.

In this work, we consider the case when $\phi$ is the identity operator on $L^{2}(\mathbb{T})$, that is, we take the additive noise to be the space-time white noise $\partial^{2} B / \partial t \partial x$, where $B(x, t)$ is a two parameter Brownian motion on $\mathbb{T} \times \mathbb{R}^{+}$. Note that $\phi$ is a Hilbert-Schmidt operator from $L^{2}(\mathbb{T})$ to $H^{s}(\mathbb{T})$ for $s<-\frac{1}{2}$ but not for $s \geq-\frac{1}{2}$.

MSC2000: 35Q53, 60H15.

Keywords: stochastic KdV, white noise, local well-posedness. 
Suppose that $u$ is the solution to (1), or equivalently to (2) with $\phi=\mathrm{Id}$, the identity operator on $L^{2}(\mathbb{T})$. Let $v_{1}(x, t)=u\left(x+\alpha_{0} t, t\right)-\alpha_{0}$, where $\alpha_{0}$ is the mean of $u_{0}$. Then, $v_{1}$ satisfies (1) with the mean-zero initial condition $u_{0}-\alpha_{0}$. Now, let $\mathbb{P}_{0}$ be the projection onto the spatial frequency 0 , and $\mathbb{P}_{n \neq 0}=\mathrm{Id}-\mathbb{P}_{0}$. Note that $\mathbb{P}_{0} W(t)=\beta_{0}(t) e_{0}(x)=\frac{1}{\sqrt{2 \pi}} \beta_{0}(t)$. By letting $v_{2}=v_{1}-\frac{1}{\sqrt{2 \pi}} \beta_{0}(t)$, we see that $u$ satisfies (1) if and only if $v_{2}$ satisfies

$$
\left\{\begin{array}{l}
d v_{2}+\left(\partial_{x}^{3} v_{2}+\left(v_{2}+\frac{1}{\sqrt{2 \pi}} \beta_{0}(t)\right) \partial_{x} v_{2}\right) d t=\mathbb{P}_{n \neq 0} d W, \\
v_{2}(x, 0)=u_{0}(x)-\alpha_{0}
\end{array}\right.
$$

almost surely since $\beta_{0}(0)=0$ a.s. By setting $v_{3}(x, t)=v_{2}\left(x+c_{\omega}(t), t\right)$ with $c_{\omega}(t)=\int_{0}^{t} \frac{1}{\sqrt{2 \pi}} \beta_{0}\left(t^{\prime}\right) d t^{\prime}$, it follows that $v_{3}$ satisfies

$$
\left\{\begin{array}{l}
d v_{3}+\left(\partial_{x}^{3} v_{3}+v_{3} \partial_{x} v_{3}\right) d t=d \widetilde{W} \\
v_{3}(x, 0)=u_{0}(x)-\alpha_{0}
\end{array}\right.
$$

where

$$
\widetilde{W}(x, t)=\sum_{n \neq 0} \frac{1}{\sqrt{2}} \beta_{n}(t) e_{n}\left(x+c_{\omega}(t)\right)=\sum_{n \neq 0} \frac{1}{\sqrt{2}} \beta_{n}(t) e^{i n c_{\omega}(t)} e_{n}(x) ;
$$

that is, $v_{3}$ solves (2), where

$$
\phi=\operatorname{diag}\left(\phi_{n} ; n \neq 0\right) \quad \text { with } \quad \phi_{n}(t)=e^{i n c_{\omega}(t)} \quad \text { and } \quad c_{\omega}(t)=\int_{0}^{t} \frac{1}{\sqrt{2 \pi}} \beta_{0}\left(t^{\prime}\right) d t^{\prime}
$$

(with respect to the basis $\left\{e_{n}\right\}_{n \in \mathbb{Z}}$ ). Moreover, $v_{3}$ has spatial mean 0 (as long as it exists) since $e_{0}$ does not lie in the range of $\phi$. Therefore, in the remainder of the paper, we concentrate on studying the local well-posedness of (2) with $\phi$ given by (3) and the mean-zero initial condition $u_{0}$ (which implies that $u$ has spatial mean 0 as long as it exists).

Recall that $u$ is called a (local-in-time) mild solution to (2) if $u$ satisfies

$$
u(t)=S(t) u_{0}-\frac{1}{2} \int_{0}^{t} S\left(t-t^{\prime}\right) \partial_{x} u^{2}\left(t^{\prime}\right) d t^{\prime}+\int_{0}^{t} S\left(t-t^{\prime}\right) \phi\left(t^{\prime}\right) d W\left(t^{\prime}\right)
$$

at least for $t \in[0, T]$ for some $T>0$, where $S(t)=e^{-t \partial_{x}^{3}}$.

Note that the first two terms in (4) also appear in the deterministic KdV theory. Thus, we briefly review recent well-posedness results of the periodic (deterministic) KdV.

$$
\left\{\begin{array}{l}
u_{t}+u_{x x x}+u u_{x}=0, \\
\left.u\right|_{t=0}=u_{0},
\end{array} \quad(x, t) \in \mathbb{T} \times \mathbb{R} .\right.
$$

Bourgain [1993] introduced a new weighted space-time Sobolev space $X^{s, b}$ whose norm is given by

$$
\|u\|_{X^{s, b}(\mathbb{T} \times \mathbb{R})}=\left\|\langle n\rangle^{s}\left\langle\tau-n^{3}\right\rangle^{b} \hat{u}(n, \tau)\right\|_{L_{n, \tau}^{2}(\mathbb{Z} \times \mathbb{R})},
$$

where $\langle\cdot\rangle=1+|\cdot|$. He proved the local well-posedness of (5) in $L^{2}(\mathbb{T})$ via the fixed point argument, immediately yielding the global well-posedness in $L^{2}(\mathbb{T})$ thanks to the conservation of the $L^{2}$ norm. Kenig et al. [1996] improved Bourgain's result and established the local well-posedness in $H^{-\frac{1}{2}}(\mathbb{T})$ by establishing the bilinear estimate

$$
\left\|\partial_{x}(u v)\right\|_{X^{s, \frac{1}{2}}} \lesssim\|u\|_{X^{s, \frac{1}{2}}}\|v\|_{X^{s, \frac{1}{2}}}
$$


for $s \geq-\frac{1}{2}$ under the mean-zero assumption on $u$ and $v$. Colliander et al. [2003] proved the corresponding global well-posedness result via the $I$-method.

There are also results on (5) which exploit its complete integrability. Bourgain [1997] proved the global well-posedness of (5) in the class $M(\mathbb{T})$ of measures $\mu$, assuming that its total variation $\|\mu\|$ is sufficiently small. His proof is based on the trilinear estimate on the second iteration of the integral formulation of (5), assuming an a priori uniform bound on the Fourier coefficients of the solution $u$ of the form

$$
\sup _{n \in \mathbb{Z}}|\hat{u}(n, t)|<C \quad \text { for all } t \in \mathbb{R} .
$$

He then established (8) using complete integrability. More recently, Kappeler and Topalov [2006] proved the global well-posedness of the $\mathrm{KdV}$ in $H^{-1}(\mathbb{T})$ via the inverse spectral method.

There are also results on the necessary conditions on the regularity with respect to smoothness or uniform continuity of the solution map : $u_{0} \in H^{s}(\mathbb{T}) \rightarrow u(t) \in H^{s}(\mathbb{T})$. Bourgain [1997] showed that if the solution map is $C^{3}$, then $s \geq-\frac{1}{2}$. Christ et al. [2003] proved that if the solution map is uniformly continuous, then $s \geq-\frac{1}{2}$. (See also [Kenig et al. 2001].) These results, in particular, imply that we cannot hope to have a local-in-time solution of $\mathrm{KdV}$ via the fixed point argument in $H^{s}, s<-\frac{1}{2}$. Recall that, for each fixed $t$, the space-time white noise $\partial^{2} B / \partial t \partial x$ lies in $\bigcap_{s<-1 / 2} H^{s} \backslash H^{-\frac{1}{2}}$ almost surely. Hence, these results for $\mathrm{KdV}$ cannot be applied to study the local well-posedness of (1).

Now, let us discuss the spaces which capture the regularities of the spatial and space-time white noise. Recently, we proved the invariance of the (spatial) white noise for the (deterministic) KdV in [Oh 2009a] (also see [Oh 2009b]) by first establishing the local well-posedness in an appropriate Banach space containing the support of the (spatial) white noise. Define the Besov-type space via the norm

$$
\|f\|_{\hat{b}_{p, \infty}^{s}}:=\|\hat{f}\|_{b_{p, \infty}^{s}}=\sup _{j}\left\|\langle n\rangle^{s} \hat{f}(n)\right\|_{L_{|n| \sim 2^{j}}^{p}}=\sup _{j}\left(\sum_{|n| \sim 2^{j}}\langle n\rangle^{s p}|\hat{f}(n)|^{p}\right)^{1 / p} .
$$

In [Oh 2009a], using the theory of abstract Wiener spaces, we showed that $\hat{b}_{p, \infty}^{s}$ contains the full support of the (spatial) white noise for $s p<-1$. (The statement also holds true for $s p=-1$.)

Let's consider the stochastic convolution $\Phi(t)$ given by

$$
\Phi(t)=\int_{0}^{t} S\left(t-t^{\prime}\right) \phi\left(t^{\prime}\right) d W\left(t^{\prime}\right)
$$

where $\phi$ is given by (3). Define a variant of the $X^{s, b}$ space adjusted to $\hat{b}_{p, \infty}^{s}(\mathbb{T})$. Let $X_{p, q}^{s, b}$ be the completion of the Schwartz class $\mathscr{Y}(\mathbb{T} \times \mathbb{R})$ under the norm

$$
\|u\|_{X_{p, q}^{s, b}}=\left\|\langle n\rangle^{s}\left\langle\tau-n^{3}\right\rangle^{b} \hat{u}(n, \tau)\right\|_{b_{p, \infty}^{0} L_{\tau}^{q}} .
$$

Note that $X_{p, q}^{s, b}$ defined in (11) is the space of functions $u$ such that $S(-t) u(\cdot, t) \in\left(\hat{b}_{p, \infty}^{s}\right)_{x}\left(\mathscr{F} L^{b, q}\right)_{t}$, where $\mathscr{F} L^{b, q}$ is defined via the norm

$$
\|f\|_{\mathscr{F} L^{b, q}}:=\left\|\langle\tau\rangle^{b} \hat{f}(\tau)\right\|_{L^{q}} .
$$

In the same paper we also showed that the local-in-time white noise is supported on $\mathscr{F} L^{c, q}$ for $c q<-1$. This implies that the Brownian motion belongs locally in time to $\mathscr{F} L^{b, q}$ for $(b-1) q<-1$. Hence, with 
$b<\frac{1}{2}$ and $q=2$, we see that the local-in-time stochastic convolution $\eta(t) \Phi(t)$ lies in $X_{p, q}^{s, b}$ almost surely, with $s p<-1, b<\frac{1}{2}$ and $q=2$, where $\eta(t)$ is a smooth cutoff supported on $[-1,2]$ with $\eta(t) \equiv 1$ on $[0,1]$.

The argument in [De Bouard et al. 2004] is based on the result by Roynette [1993] on the endpoint regularity of the Brownian motion, which states that the Brownian motion $\beta(t)$ belongs to the Besov space $B_{p, q}^{1 / 2}$ if and only if $q=\infty$ (with $1 \leq p<\infty$ ). The authors then proved a variant of the bilinear estimate (7) by Kenig, Ponce and Vega, adjusted to their Besov space setting, establishing the local wellposedness via the fixed point theorem. The use of a variant of the bilinear estimate (7) required a slight regularization of the noise in space via $\phi$ so that the smoothed noise has the spatial regularity $s>-\frac{1}{2}$. Thus, they could not treat the space-time white noise, that is, $\phi=$ Id.

Our result is based on two observations. First, our $l_{n}^{p}$-based function spaces $\hat{b}_{p, \infty}^{s}$ in (9) and $X_{p, q}^{s, b}$ in (11) capture the regularity of the spatial and space-time white noise for $s p<-1, b<\frac{1}{2}$ and $q=2$. The second is that we can indeed carry out the argument in [Bourgain 1997], a nonlinear estimate on the second iteration, without assuming the a priori bound (8), if we take the initial data $u_{0} \in \hat{b}_{p, \infty}^{s}$ for $s>-\frac{1}{2}$ with $p>2$. Then, we construct a solution $u$ as a strong limit of the smooth solutions $u^{N}$ (with smooth $u_{0}^{N}$ and $\phi^{N}$ ) of (2). Note that our nonlinear estimate on the second iteration in Section 5 depends on the stochastic term, whereas the bilinear estimate in [De Bouard et al. 2004] is entirely deterministic.

Finally, we present our main results.

Theorem 1. Let $\phi$ be as in (3) and $p=2+$. Let $s=-\frac{1}{2}+\delta$ with $(p-2) /(4 p)<\delta<(p-2) /(2 p)$, so $s p<-1$. Also, let $u_{0}$ be $\mathscr{F}_{0}$-measurable such that it has mean 0 and belongs to $\hat{b}_{p, \infty}^{s}(\mathbb{T})$ almost surely. Then, there exists a stopping time $T_{\omega}>0$ and a unique process $u \in C\left(\left[0, T_{\omega}\right] ; \hat{b}_{p, \infty}^{s}(\mathbb{T})\right)$ satisfying (2) on $\left[0, T_{\omega}\right]$ almost surely.

As a corollary, we obtain:

Theorem 2. The stochastic $K d V$ (1) with the additive space-time white noise is locally well posed almost surely (with the prescribed mean on $u_{0}$ ).

Remark 1.1. Our argument provides an answer to the question posed in [Bourgain 1997, remark on p. 120], at least in the local-in-time setting. The deterministic part of the nonlinear estimate in Section 5 can be used to establish the local well-posedness of (5) for a finite Borel measure $u_{0}=\mu \in M(\mathbb{T})$ with $\|\mu\|<\infty$ without the complete integrability or the smallness assumption on $\mu$. Note that $\mu \in \hat{b}_{p, \infty}^{s}$ for $s p \leq-1$ since $\sup _{n}|\widehat{\mu}(n)|<\|\mu\|<\infty$. Hence, it can be used to study the Cauchy problem on $M(\mathbb{T})$ for nonintegrable KdV-variants. Also, see [Oh 2009b].

Remark 1.2. Let $\mathscr{F} L^{s, p}(\mathbb{T})$ be the space of functions on $\mathbb{T}$ defined via the norm $\|f\| \mathscr{F} L^{s, p}=\left\|\langle n\rangle^{s} \hat{f}(n)\right\|_{L_{n}^{p}}$. Recall from [Oh 2009a] that $\mathscr{F} L^{s, p}(\mathbb{T})$ contains the support of the (spatial) white noise when $s p<-1$. Then, Theorems 1 and 2 can also be established in $\mathscr{F} L^{s, p}(\mathbb{T})$ for $s=-\frac{1}{2}+, p=2+$ with $s p<-1$. The modification is straightforward once we note that $\|f\|_{\mathscr{F} L^{s-\varepsilon, p}} \lesssim\|f\|_{\hat{b}_{p, \infty}^{s}}$ for any $\varepsilon>0$, and thus we omit the details.

This paper is organized as follows: In Section 2, we introduce some notations. In Section 3, we introduce function spaces along with their embeddings and state deterministic linear estimates from [Bourgain 1993] and [Oh 2009a]. In Section 4, we study some basic properties of the stochastic convolution. In Section 5, we prove Theorem 1 by establishing the nonlinear estimate on the second iteration of the integral formulation (4). 


\section{Notation}

In the periodic setting on $\mathbb{T}$, the spatial Fourier domain is $\mathbb{Z}$. Let $d n$ be the normalized counting measure on $\mathbb{Z}$. We say that $f \in L^{p}(\mathbb{Z})$, where $1 \leq p<\infty$, if

$$
\|f\|_{L^{p}(\mathbb{Z})}=\left(\int_{\mathbb{Z}}|f(n)|^{p} d n\right)^{1 / p}:=\left(\frac{1}{2 \pi} \sum_{n \in \mathbb{Z}}|f(n)|^{p}\right)^{1 / p}<\infty .
$$

If $p=\infty$, we have the obvious definition involving the supremum. We often drop $2 \pi$ for simplicity. If a function depends on both $x$ and $t$, we use ${ }^{\wedge_{x}}$ (and ${ }^{\wedge_{t}}$ ) to denote the spatial (and temporal) Fourier transform, respectively. However, when there is no confusion, we simply use ${ }^{\wedge}$ to denote the spatial Fourier transform, the temporal Fourier transform, and the space-time Fourier transform, depending on the context.

For a Banach space $X \subset \mathscr{Y}^{\prime}(\mathbb{T} \times \mathbb{R})$, we use $\hat{X}$ to denote the space of the Fourier transforms of the functions in $X$, which is a Banach space with the norm $\|f\|_{\hat{X}}=\left\|\mathscr{F}_{n, \tau}^{-1} f\right\|_{X}$, where $\mathscr{F}^{-1}$ denotes the inverse Fourier transform (in $n$ and $\tau$ ). Also, for a space $Y$ of functions on $\mathbb{Z}$, we use $\hat{Y}$ to denote the space of the inverse Fourier transforms of the functions in $Y$ with the norm $\|f\|_{\hat{Y}}=\|\mathscr{F} f\|_{Y}$. Now, define $\hat{b}_{p, q}^{s}(\mathbb{T})$ by the norm

$$
\|f\|_{\hat{b}_{p, q}^{s}(\mathbb{T})}=\|\hat{f}\|_{b_{p, q}^{s}(\mathbb{Z})}:=\|\|\langle n\rangle^{s} \hat{f}(n)\left\|_{L_{|n| \sim 2^{j}}^{p}}\right\|_{l_{j}^{q}}=\left(\sum_{j=0}^{\infty}\left(\sum_{|n| \sim 2^{j}}\langle n\rangle^{s p}|\hat{f}(n)|^{p}\right)^{q / p}\right)^{1 / q}
$$

for $q<\infty$ and by (9) when $q=\infty$.

Throughout the paper, $\eta(t)$ denotes a smooth cutoff supported on $[-1,2]$ with $\eta(t) \equiv 1$ on $[0,1]$, and let $\eta_{T}(t)=\eta\left(T^{-1} t\right)$. We use $c, C$ to denote various constants, usually depending only on $s, p$, and $\delta$. If a constant depends on other quantities, we make it explicit. We use $A \lesssim B$ to denote an estimate of the form $A \leq C B$. Similarly, we use $A \sim B$ to denote $A \lesssim B$ and $B \lesssim A$ and use $A \ll B$ when there is no general constant $C$ such that $B \leq C A$. We also use $a+$ and $a-$ to denote $a+\varepsilon$ and $a-\varepsilon$, respectively, for arbitrarily small $\varepsilon \ll 1$.

\section{Function spaces and basic embeddings}

Let $X^{s, b}$ denote the usual periodic Bourgain space defined in (6). We often use the shorthand notation $\|\cdot\|_{s, b}$ to denote the $X^{s, b}$ norm. Now, define $X_{p, q}^{s, b}$, the Bourgain space adapted to $\hat{b}_{p, \infty}^{s}$, to be the completion of the Schwartz functions on $\mathbb{T} \times \mathbb{R}$ with respect to the norm given by

$$
\|u\|_{X_{p, q}^{s, b}}=\left\|\langle n\rangle^{s}\left\langle\tau-n^{3}\right\rangle^{b} \hat{u}(n, \tau)\right\|_{b_{p, \infty}^{0} L_{\tau}^{q}}=\sup _{j}\left\|\langle n\rangle^{s}\left\langle\tau-n^{3}\right\rangle^{b} \hat{u}(n, \tau)\right\|_{L_{|n| \sim 2^{j}}^{p} L_{\tau}^{q}} .
$$

In the following, we take $p=2+$ and $s=-\frac{1}{2}+=-\frac{1}{2}+\delta$ with $\delta<(p-2) / 2 p$ (and $\delta>(p-2) / 4 p$ ) such that $s p<-1$. Lastly, given $T>0$, we define $X_{p, q}^{s, b, T}$ as a restriction of $X_{p, q}^{s, b}$ on $[0, T]$ by

$$
\|u\|_{X_{p, q}^{s, b, T}}=\|u\|_{X_{p, q}^{s, b}[0, T]}=\inf \left\{\|\widetilde{u}\|_{X_{p, q}^{s, b}}:\left.\widetilde{u}\right|_{[0, T]}=u\right\} .
$$

We define the local-in-time versions of the other function spaces analogously. 
Now, we discuss the basic embeddings. For $p \geq 2$, we have $\left\|a_{n}\right\|_{L_{n}^{p}} \leq\left\|a_{n}\right\|_{L_{n}^{2}}$. Thus, we have $\|f\|_{\hat{b}_{p, \infty}^{s}} \leq\|f\|_{H^{s}}$, and thus

$$
\|u\|_{X_{p, 2}^{s, b}} \leq\|u\|_{X^{s, b}}
$$

By the Hölder inequality, we have

$$
\begin{aligned}
\|f\|_{H^{-\frac{1}{2}-\delta}} & =\left(\sum_{j}\left(2^{j}\right)^{0-}\left\|\langle n\rangle^{-\frac{1}{2}-\delta+} \hat{f}(n)\right\|_{|n| \sim 2^{j}}^{2}\right)^{1 / 2} \\
& \leq \sup _{j}\left\|\langle n\rangle^{-2 \delta+}\right\|_{L^{2 p / p-2}}\left\|\langle n\rangle^{-\frac{1}{2}+\delta} \hat{f}(n)\right\|_{L_{n}^{p}} \leq\|f\|_{\hat{b}_{p, \infty}^{s}}
\end{aligned}
$$

for $s=-\frac{1}{2}+\delta$ with $\delta>(p-2) / 4 p$. Hence, for $s=-\frac{1}{2}+\delta$ with $\delta>(p-2) / 4 p$, we have

$$
\|u\|_{X^{-\frac{1}{2}-\delta, b}} \lesssim\|u\|_{X_{p, 2}^{s, b}}
$$

Now, we briefly go over the linear estimates. Let $S(t)=e^{-t \partial_{x}^{3}}$ and $T \leq 1$ in the following. We first present the homogeneous and nonhomogeneous linear estimates. See [Bourgain 1993; Kenig et al. 1993; Oh 2009a] for details of the proofs.

Lemma 3.1. For any $s \in \mathbb{R}$ and $b<\frac{1}{2}$, we have $\left\|S(t) u_{0}\right\|_{X_{p, 2}^{s, b, T}} \lesssim T^{(1 / 2)-b}\left\|u_{0}\right\|_{\hat{b}_{p, \infty}^{s}}$.

Lemma 3.2. For any $s \in \mathbb{R}$ and $b \leq \frac{1}{2}$, we have

$$
\left\|\int_{0}^{t} S\left(t-t^{\prime}\right) F\left(x, t^{\prime}\right) d t^{\prime}\right\|_{X_{p, 2}^{s, b, T}} \lesssim\|F\|_{X_{p, 2}^{s, b-1}}+\|F\|_{X_{p, 1}^{s,-1}} .
$$

Also, we have $\left\|\int_{0}^{t} S\left(t-t^{\prime}\right) F\left(x, t^{\prime}\right) d t^{\prime}\right\|_{X_{p, 2}^{s, b},} \lesssim\|F\|_{X_{p, 2}^{s, b-1}}$ for $b>\frac{1}{2}$.

The next lemma is the periodic $L^{4}$ Strichartz estimate due to Bourgain [1993].

Lemma 3.3. Let $u$ be a function on $\mathbb{T} \times \mathbb{R}$. Then, we have $\|u\|_{L_{x, t}^{4}} \lesssim\|u\|_{X^{0, \frac{1}{3}}}$.

Lastly, recall that by restricting the Bourgain spaces onto a small time interval $[0, T]$, we can gain a small power of $T$. See [Colliander and Oh 2009] for the proof.

Lemma 3.4. For $0 \leq b^{\prime}<b \leq \frac{1}{2}$, we have

$$
\|u\|_{X^{s, b^{\prime}, T}}=\left\|\eta_{T} u\right\|_{X^{s, b^{\prime}, T}} \lesssim T^{b-b^{\prime}-}\|u\|_{X^{s, b}} .
$$

\section{Stochastic convolution}

In this section, we study basic properties of the stochastic convolution $\Phi(t)$ defined in (10). In particular, we prove that $\eta \Phi$ belongs to $X_{p, 2}^{s, b, T}$ and is continuous from $[0, T]$ into $\hat{b}_{p, \infty}^{s}$ for $T \leq 1$ almost surely for $s p<-1$ and $(b-1) \cdot 2<-1$, where $\eta(t)$ is a smooth cutoff supported on $[-1,2]$ with $\eta(t) \equiv 1$ on $[0,1]$.

Before stating the main results, we point out the following. Let $\phi$ be the identity operator on $L^{2}(\mathbb{T})$ or be as in (3). Then, we know that such $\phi$ is Hilbert-Schmidt from $L^{2}(\mathbb{T})$ into $H^{s}(\mathbb{T})$ if and only if $s<-\frac{1}{2}$. In other words, with a slight abuse of notation, define

$$
\phi:=\sum_{n \in \mathbb{Z}} \phi e_{n}=\sum_{n \in \mathbb{Z}} \phi_{n} e_{n}
$$


in view of $\phi=\operatorname{diag}\left(\phi_{n} ; n \neq 0\right)$. Then, we have $\phi \in H^{s}(\mathbb{T})$ if and only if $s<-\frac{1}{2}$. Moreover, we have $\|\phi\|_{H S\left(L^{2} ; H^{s}\right)}=\|\phi\|_{H^{s}}$, where $\|\cdot\|_{H S\left(L^{2} ; H^{s}\right)}$ denotes the Hilbert-Schmidt norm from $L^{2}(\mathbb{T})$ to $H^{s}(\mathbb{T})$. For such $\phi$, we also have $\phi \in \hat{b}_{p, \infty}^{s}(\mathbb{T})$ if and only if $s p \leq-1$, and we can use $\|\phi\|_{\hat{b}_{p, \infty}^{s}}$ to discuss the regularity of $\phi$ in place of the Hilbert-Schmidt norm. This is one of the reasons for using this space. (We need only $s p<-1$ for our purpose since the nonlinear estimate in Section 5 holds for $s=-\frac{1}{2}$ and $p=2+$ with $s p<-1$.)

Proposition 4.1. Let $0<T \leq 1$ and $p=2+$. Let $s=-\frac{1}{2}+\delta$ and $b=\frac{1}{2}-\delta$, with $(p-2) / 4 p<\delta<(p-2) / 2 p$ such that $s p<-1$ and $(b-1) \cdot 2<-1$. Then, for the stochastic convolution $\Phi(t)$ defined in $(10)$ with $\phi$ as in (3), we have

In particular, $\Phi \in X_{p, 2}^{-\frac{1}{2}+\delta, \frac{1}{2}-\delta, T}$ almost surely.

$$
\mathbb{E}\left[\|\eta \Phi\|_{X_{p, 2}^{s, b}, T}\right] \leq C(\eta, s, p)<\infty .
$$

Before going into the proof of Proposition 4.1, recall the following. Let $\beta_{1}$ and $\beta_{2}$ be independent realvalued Brownian motions on $(\Omega, \mathscr{F}, P)$, and $f_{1}(t, \omega)$ and $f_{2}(t, \omega)$ be real-valued stochastic processes independent of $\beta_{1}$ and $\beta_{2}$. Then, we can regard $\beta_{j}$ and $f_{j}$ as $\beta_{j}(t, \omega)=\beta_{j}\left(t, \omega_{1}\right)$ and $f_{j}(t, \omega)=f_{j}\left(t, \omega_{2}\right)$, where $\omega=\left(\omega_{1}, \omega_{2}\right) \in \Omega_{1} \times \Omega_{2}=\Omega$. Thus, in taking an expectation, we can first integrate over $\omega_{1} \in \Omega_{1}$. Then, for $m \in \mathbb{N}$, we have

$$
\begin{aligned}
\mathbb{E}\left[\mid \int_{a}^{b} f_{1}(t) d \beta_{1}(t)\right. & \left.+\left.\int_{a}^{b} f_{2}(t) d \beta_{2}(t)\right|^{2 m}\right] \\
& =\mathbb{E}\left[\sum_{k=0}^{2 m}\left(\begin{array}{c}
2 m \\
k
\end{array}\right)\left(\int_{a}^{b} f_{1}(t) d \beta_{1}(t)\right)^{k}\left(\int_{a}^{b} f_{2}(t) d \beta_{2}(t)\right)^{2 m-k}\right] \\
& =\mathbb{E}_{\Omega_{2}}\left[\sum_{n=0}^{m}\left(\begin{array}{c}
2 m \\
2 n
\end{array}\right) \frac{(2 n) !}{2^{n} n !}\left\|f_{1}\left(\cdot, \omega_{2}\right)\right\|_{L^{2}(a, b)}^{2 n} \frac{(2(m-n)) !}{2^{m-n}(m-n) !}\left\|f_{2}\left(\cdot, \omega_{2}\right)\right\|_{L^{2}(a, b)}^{2(m-n)}\right] .
\end{aligned}
$$

In the computation above, we used the fact that, for each fixed $\omega_{2}, \int_{a}^{b} f_{j}\left(t, \omega_{2}\right) d \beta_{j}\left(t, \omega_{1}\right)$ is a Gaussian random variable on $\Omega_{1}$ with variance $\left\|f_{j}\left(\cdot, \omega_{2}\right)\right\|_{L^{2}(a, b)}^{2}$.

Proof. By the Hölder inequality, we have

$$
\left\|\left\langle\tau-n^{3}\right\rangle^{(1 / 2)-\delta} \hat{u}(n, \tau)\right\|_{L_{\tau}^{2}} \leq\left\|\left\langle\tau-n^{3}\right\rangle^{-2 \delta}\right\|_{L_{\tau}^{2 p / p-2}}\left\|\left\langle\tau-n^{3}\right\rangle^{(1 / 2)+\delta} \hat{u}(n, \tau)\right\|_{L_{\tau}^{p}},
$$

that is, we have $\|\eta \Phi\|_{X_{p, 2}^{s, 12-\delta}} \lesssim\|\eta \Phi\|_{X_{p, p}^{s, 1 / 2+\delta}}$ as long as $\delta>(p-2) / 4 p$. Thus, we will work in $X_{p, p}^{s, 1 / 2+\delta}$ in the following.

Define $g(t)=\eta(t) \int_{0}^{t} S(-r) \phi(r) d W(r)$ such that $\eta(t) \Phi(\cdot, t)=S(t) g(\cdot, t)$. Assume that each $\beta_{n}$ is extended to a Brownian motion on $\mathbb{R}$ in such a way that the family $\left\{\beta_{n}\right\}_{n \geq 0}$ is still independent. Note that for $t \in[0, T]$, we have

$$
\hat{g}(n, t)=\eta(t) \int_{0}^{t} \eta(r) e^{-i r n^{3}} \phi_{n}(r) \chi_{[0, T]}(r) \frac{1}{\sqrt{2}} d \beta_{n}(r) .
$$

We have inserted $\eta(r)$ and $\chi_{[0, T]}(r)$ in the integrand since $\eta(r) \chi_{[0, T]}(r) \equiv 1$ for $r \in[0, t] \subset[0, T]$. For notational simplicity, we use $\phi_{n}(r)$ to denote $\phi_{n}(r) \chi_{[0, T]}(r)$ in the following, that is, we assume that $\phi_{n}$ is supported on $[0, T]$. By (3), we have $\left|\phi_{n}(r)\right| \leq 1$ for $r \in \mathbb{R}$. 
Now, we write the left-hand side of (19) as

$$
\begin{aligned}
& \mathbb{E}\left[\|\eta \Phi\|_{\substack{X_{p, p}^{s, \frac{1}{2}+\delta, T}\\
}}\right] \mathbb{E}\left[\sup _{j} 2^{j s}\left(\sum_{|n| \sim 2^{j}} \sum_{k=1}^{\infty} 2^{k p\left(\frac{1}{2}+\delta\right)} \int_{|\tau| \sim 2^{k}}|\hat{g}(n, \tau)|^{p} d \tau\right)^{1 / p}\right] \\
& +\mathbb{E}\left[\sup _{j} 2^{j s}\left(\sum_{|n| \sim 2^{j}} \int_{|\tau| \leq 2}|\hat{g}(n, \tau)|^{p} d \tau\right)^{1 / p}\right] .
\end{aligned}
$$

Part 1. First, we estimate the second term in (22). Let

$$
G_{n}(r, \tau)=\eta(r) e^{-i r n^{3}} \phi_{n}(r) \int_{r}^{\infty} \eta(t) e^{-i t \tau} d t .
$$

Also write $\beta_{n}=\beta_{n}^{(r)}+i \beta_{n}^{(i)}$ where $\beta_{n}^{(r)}=\operatorname{Re} \beta_{n}$ and $\beta_{n}^{(i)}=\operatorname{Im} \beta_{n}$. Then, by the stochastic Fubini Theorem, we have, for $m \in \mathbb{N}$,

$$
\begin{aligned}
& \mathbb{E}\left[|\hat{g}(n, \tau)|^{2 m}\right]=\mathbb{E}\left[\left|\int_{\mathbb{R}} \eta(t) e^{-i t \tau} \int_{-\infty}^{t} \eta(r) e^{-i r n^{3}} \phi_{n}(r) \frac{1}{\sqrt{2}} d \beta_{n}(r) d t\right|^{2 m}\right] \\
&=2^{-m} \mathbb{E}\left[\left|\int_{-1}^{2} G_{n}(r, \tau) d \beta_{n}(r)\right|^{2 m}\right] \\
& \lesssim \mathbb{E}\left[\left|\int_{-1}^{2} \operatorname{Re} G_{n}(r, \tau) d \beta_{n}^{(r)}(r)-\int_{-1}^{2} \operatorname{Im} G_{n}(r, \tau) d \beta_{n}^{(i)}(r)\right|^{2 m}\right] \\
&+\mathbb{E}\left[\left|\int_{-1}^{2} \operatorname{Im} G_{n}(r, \tau) d \beta_{n}^{(r)}(r)+\int_{-1}^{2} \operatorname{Re} G_{n}(r, \tau) d \beta_{n}^{(i)}(r)\right|^{2 m}\right]
\end{aligned}
$$

Note that $\left|\operatorname{Re} G_{n}(r, \tau)\right|,\left|\operatorname{Im} G_{n}(r, \tau)\right| \leq\left|G_{n}(r, \tau)\right| \leq\|\eta\|_{L^{1}}\left|\phi_{n}(r)\right| \lesssim\|\eta\|_{L^{1}} \chi_{[0, T]}(r)$. Thus, we have

$$
\left\|\operatorname{Re} G_{n}(r, \tau)\right\|_{L_{r}^{2}}^{2 k}\left\|\operatorname{Im} G_{n}(r, \tau)\right\|_{L_{r}^{2}}^{2(m-k)} \lesssim\|\eta\|_{L^{1}}^{2 m}
$$

for $k=0, \ldots, m$. Then, by (20) along with the independence of $\phi_{n}, \beta_{n}^{(r)}$, and $\beta_{n}^{(i)}$, we have

$$
\|\hat{g}(n, \tau)\|_{L^{2 m}(\Omega)} \leq C=C(\eta, m)
$$

independent of $n$ and $\tau$. Hence, for $p \in(2,4)$, we have

$$
\left(\mathbb{E}\left[|\hat{g}(n, \tau)|^{p}\right]\right)^{1 / p} \leq\|\hat{g}(n, \tau)\|_{L^{2}(\Omega)}^{\theta}\|\hat{g}(n, \tau)\|_{L^{4}(\Omega)}^{1-\theta} \lesssim 1
$$

by interpolation, where $\theta \in(0,1)$ such that $\frac{1}{p}=\frac{\theta}{2}+\frac{1-\theta}{4}$. Then, the second term in (22) is estimated by

$$
\begin{aligned}
(22) & \leq\left(\sum_{j=0}^{\infty} 2^{j s p} \sum_{|n| \sim 2^{j}} \int_{|\tau| \leq 2} \mathbb{E}\left[|\hat{g}(n, \tau)|^{p}\right] d \tau\right)^{1 / p} \\
& \lesssim\left(\sum_{j=0}^{\infty} 2^{j s p} \sum_{|n| \sim 2^{j}} 1\right)^{1 / p} \sim\left(\sum_{j=0}^{\infty} 2^{(s p+1) j}\right)^{1 / p} \leq C<\infty,
\end{aligned}
$$

since $s p<-1$. 
Part 2. Next, we estimate the first term in (22). Let

$$
\left\{\begin{array}{l}
G_{n}^{(1)}(r, \tau)=\eta(r) e^{-i r n^{3}} \phi_{n}(r) \int_{r}^{\infty} \eta^{\prime}(t)\left(e^{-i t \tau} / i \tau\right) d t \\
G_{n}^{(2)}(r, \tau)=\eta^{2}(r) e^{-i r n^{3}} \phi_{n}(r)\left(e^{-i r \tau} / i \tau\right)
\end{array}\right.
$$

Then, by the stochastic Fubini theorem and integration by parts, we have

$$
\begin{aligned}
\sqrt{2} \hat{g}(n, \tau) & =\int_{-1}^{2} G_{n}(r, \tau) d \beta_{n}(r)=\int_{-1}^{2} G_{n}^{(1)}(r, \tau) d \beta_{n}(r)+\int_{-1}^{2} G_{n}^{(2)}(r, \tau) d \beta_{n}(r) \\
& =: I_{n}^{(1)}(\tau)+I_{n}^{(2)}(\tau) .
\end{aligned}
$$

Thus $|\hat{g}(n, \tau)|^{p} \lesssim\left|I_{n}^{(1)}(\tau)\right|^{p}+\left|I_{n}^{(2)}(\tau)\right|^{p}$

First, we estimate the contribution from $G_{n}^{(1)}$. For $|\tau| \sim 2^{k}$, we have

$$
\left|\int_{r}^{\infty} \eta^{\prime}(t) \frac{e^{-i t \tau}}{i \tau} d t\right| \leq\left|\tau^{-2} \eta^{\prime}(r)\right|+\left|\int_{r}^{\infty} \eta^{\prime \prime}(t) \frac{e^{-i t \tau}}{\tau^{2}} d t\right| \leq C_{\eta} 2^{-2 k}
$$

by partial integration. Thus, we have $\left|G_{n}^{(1)}(r, \tau)\right| \lesssim 2^{-2 k}$. Then, repeating a similar computation as in Part 1, we obtain

$$
\left(\mathbb{E}\left[\left|I_{n}^{(1)}(\tau)\right|^{p}\right]\right)^{1 / p} \leq\left\|I_{n}^{(1)}(\tau)\right\|_{L^{2}(\Omega)}^{\theta}\left\|I_{n}^{(1)}(\tau)\right\|_{L^{4}(\Omega)}^{1-\theta} \lesssim 2^{-2 k}
$$

by (20) and interpolation. Hence, the contribution to (22) is estimated by

$$
\begin{aligned}
(22) & \leq\left(\sum_{j=0}^{\infty} 2^{j s p} \sum_{|n| \sim 2^{j}} \sum_{k=1}^{\infty} 2^{k p\left(\frac{1}{2}+\delta\right)} \int_{|\tau| \sim 2^{k}} \mathbb{E}\left[\left|I_{n}^{(1)}(\tau)\right|^{p}\right] d \tau\right)^{1 / p} \\
& \lesssim\left(\sum_{j=0}^{\infty} 2^{j(s p+1)} \sum_{k=1}^{\infty} 2^{k(-(3 p / 2)+\delta p+1)}\right)^{1 / p} \leq C<\infty,
\end{aligned}
$$

since $s p<-1$ and $-\frac{3}{2} p+\delta p+1<0$.

Now, we consider the contribution from $I_{n}^{(2)}(\tau)$. With $\beta_{n}=\beta_{n}^{(r)}+i \beta_{n}^{(i)}$, we have $\left|I_{n}^{(2)}(\tau)\right|^{2} \lesssim$ $\left|\int_{-1}^{2} G_{n}^{(2)}(r, \tau) d \beta_{n}^{(r)}(r)\right|^{2}+\left|\int_{-1}^{2} G_{n}^{(2)}(r, \tau) d \beta_{n}^{(i)}(r)\right|^{2}$. We only estimate the first term since the second term is estimated in the same way. By the Ito formula (see [De Bouard et al. 2004]), we have

$$
\begin{aligned}
\left|\int_{-1}^{2} G_{n}^{(2)}(r, \tau) d \beta_{n}^{(r)}(r)\right|^{2} & =\int_{-1}^{2} \eta^{4}(t) \frac{\left|\phi_{n}(t)\right|^{2}}{\tau^{2}} d t+2 \operatorname{Re} \int_{-1}^{2} \int_{-\infty}^{t} G_{n}^{(2)}(r, \tau) d \beta_{n}^{(r)}(r) \overline{G_{n}^{(2)}}(t, \tau) d \beta_{n}^{(r)}(t) \\
& =: I_{n}^{\prime}(\tau)+I_{n}^{\prime \prime}(\tau) .
\end{aligned}
$$

The contribution from $I_{n}^{\prime}(\tau)$ is at most

$$
\begin{aligned}
(22) & \lesssim\left(\sum_{j=0}^{\infty} 2^{j s p} \sum_{|n| \sim 2^{j}} \sum_{k=1}^{\infty} 2^{k p\left(\frac{1}{2}+\delta\right)} \int_{|\tau| \sim 2^{k}}|\tau|^{-p} d \tau\left(\int_{-1}^{2} \eta^{4}(t) d t\right)^{p / 2}\right)^{1 / p} \\
& \lesssim\|\eta\|_{L^{4}}^{2}\left(\sum_{j=0}^{\infty} 2^{j(s p+1)} \sum_{k=1}^{\infty} 2^{k\left(-\frac{p}{2}+\delta p+1\right)}\right)^{1 / p} \leq C<\infty
\end{aligned}
$$

since $s p<-1$ and $\delta<(p-2) / 2 p$. 
We finally estimate the contribution from $I_{n}^{\prime \prime}(\tau)$. Write

$$
I_{n}^{\prime \prime}(\tau)=\int_{-1}^{2} H_{n}(t) d \beta_{n}^{(r)}(t),
$$

where $H_{n}(t)=\int_{-\infty}^{t} \widetilde{H}_{n}(r, t) d \beta_{n}^{(r)}(r)$ with

$$
\widetilde{H}_{n}(r, t)=2 \tau^{-2} \operatorname{Re}\left(\eta^{2}(r) \eta^{2}(t) e^{i(t-r) n^{3}} \phi_{n}(r) \overline{\phi_{n}(t)} e^{i(t-r) \tau}\right) .
$$

Then, by the Ito isometry and $\left|\phi_{n}(w, t)\right| \leq 1$ for all $(\omega, t) \in \Omega \times \mathbb{R}$, we have

$$
\begin{aligned}
\mathbb{E}\left[\left|I_{n}^{\prime \prime}(\tau)\right|^{2}\right] & =\mathbb{E}\left[\left(\int_{-1}^{2} H_{n}(t) d \beta_{n}^{(r)}(t)\right)^{2}\right] \sim \int_{-1}^{2} \mathbb{E}\left[H_{n}^{2}(t)\right] d t \\
& =\int_{-1}^{2} \mathbb{E}\left[\left(\int_{-\infty}^{t} \widetilde{H}_{n}(r, t) d \beta_{n}^{(r)}(r)\right)^{2}\right] d t=\int_{-1}^{2} \int_{-1}^{t} \mathbb{E}\left[\left|\widetilde{H}_{n}(r, t)\right|^{2}\right] d r d t \\
& \lesssim \tau^{-4} \int_{-1}^{2} \int_{-1}^{t} \eta^{4}(r) \eta^{4}(t) d r d t \lesssim \tau^{-4} .
\end{aligned}
$$

Hence, the contribution from $I_{n}^{\prime \prime}(\tau)$ is at most

$$
\begin{aligned}
(22) & \lesssim\left(\sum_{j=0}^{\infty} 2^{j s p} \sum_{|n| \sim 2^{j}} \sum_{k=1}^{\infty} 2^{k p\left(\frac{1}{2}+\delta\right)} \int_{|\tau| \sim 2^{k}} \mathbb{E}\left[\left|I_{n}^{\prime \prime}(\tau)\right|^{p / 2}\right] d \tau\right)^{1 / p} \\
& \lesssim\left(\sum_{j=0}^{\infty} 2^{j s p} \sum_{|n| \sim 2^{j}} \sum_{k=1}^{\infty} 2^{k p\left(\frac{1}{2}+\delta\right)} \int_{|\tau| \sim 2^{k}}\left(\mathbb{E}\left[\left|I_{n}^{\prime \prime}(\tau)\right|^{2}\right]\right)^{p / 4} d \tau\right)^{1 / p} \\
& \lesssim\left(\sum_{j=0}^{\infty} 2^{j(s p+1)} \sum_{k=1}^{\infty} 2^{k\left(-\frac{p}{2}+\delta p+1\right)}\right)^{1 / p} \leq C<\infty
\end{aligned}
$$

for $p \leq 4$, sp $<-1$, and $\delta<(p-2) / 2 p$.

We state a corollary to the proof of Proposition 4.1 for a general diagonal covariance operator $\phi(t, \omega)=$ $\operatorname{diag}\left(\phi_{n}(t, \omega) ; n \in \mathbb{Z}\right)$, which is independent of $\left\{\beta_{n}\right\}_{n \geq 1}$.

Corollary 4.2. Let $0<T \leq 1, p=2+$, and $s, s^{\prime} \in \mathbb{R}$ with $s<s^{\prime}$. Moreover, let $b=\frac{1}{2}-\delta$ with $(p-2) / 4 p<\delta<(p-2) / 2 p$, so $(b-1) \cdot 2<-1$. Then, for the stochastic convolution $\Phi(t)$ defined in (10) with $\phi \in L^{p}\left([0, T] \times \Omega ; \hat{b}_{p, \infty}^{s^{\prime}}\right)$, independent of $\left\{\beta_{n}\right\}_{n \geq 1}$, we have

$$
\mathbb{E}\left[\|\eta \Phi\|_{X_{p, 2}^{s, b, T}}\right] \leq C\left(\eta, s, s^{\prime}, p\right)\|\phi\|_{L^{p}\left([0, T] \times \Omega ; \hat{b}_{p, \infty}^{s^{\prime}}\right)} .
$$

In particular, $\Phi \in X_{p, 2}^{s, \frac{1}{2}-\delta, T}$ almost surely.

Proof. In the proof of Proposition 4.1, we used $\left|\phi_{n}(t)\right| \leq 1$ whenever $\phi_{n}(t)$ appeared. Now, we briefly go through that proof, keeping track of $\phi_{n}(t)$. Since $\phi$ is independent of $\left\{\beta_{n}\right\}_{n \geq 1}$, we regard $\beta_{n}$ and $\phi_{n}$ as $\beta_{n}(t, \omega)=\beta_{n}\left(t, \omega_{1}\right)$ and $\phi_{n}(t, \omega)=\phi_{n}\left(t, \omega_{2}\right)$, where $\omega=\left(\omega_{1}, \omega_{2}\right) \in \Omega_{1} \times \Omega_{2}=\Omega$. 
In (25), we have $\mathbb{E}\left[|\hat{g}(n, \tau)|^{p}\right] \lesssim \mathbb{E}_{\Omega_{2}}\left\|\phi_{n}\left(\cdot, \omega_{2}\right)\right\|_{L^{2}[0, T]}^{p}$. Then, in (26), we have

$$
\begin{aligned}
(22) & \leq\left(\sum_{j=0}^{\infty} 2^{j s p} \sum_{|n| \sim 2^{j}} \int_{|\tau| \leq 2} \mathbb{E}_{\Omega_{2}}\left\|\phi_{n}\left(\cdot, \omega_{2}\right)\right\|_{L^{2}[0, T]}^{p} d \tau\right)^{1 / p} \\
& \leq\left(\sum_{j=0}^{\infty} 2^{j\left(s-s^{\prime}\right) p} 2^{j s^{\prime} p} \sum_{|n| \sim 2^{j}}\left\|\phi_{n}\left(\cdot, \omega_{2}\right)\right\|_{L^{p}\left([0, T] \times \Omega_{2}\right)}^{p}\right)^{1 / p} \lesssim\|\phi\|_{L^{p}\left([0, T] \times \Omega ; \hat{b}_{p, \infty}^{s^{\prime}}\right)},
\end{aligned}
$$

since $s-s^{\prime}<0$. A similar modification in (30) and (31) (alternatively, (32)) takes care of the contribution from $I_{n}^{(1)}(\tau)$ (alternatively, $I_{n}^{\prime}(\tau)$ ). Now, as for $I_{n}^{\prime \prime}(\tau)$, we first integrate only over $\Omega_{1}$ in (34) and obtain

$$
\mathbb{E}_{\Omega_{1}}\left[\left|I_{n}^{\prime \prime}(\tau)\right|^{2}\right] \lesssim \tau^{-4} \int_{-1}^{2} \int_{-1}^{t} \eta^{4}(r) \eta^{4}(t)\left|\phi_{n}(r)\right|^{2}\left|\phi_{n}(t)\right|^{2} d r d t \lesssim \tau^{-4}\left\|\phi_{n}\right\|_{L^{2}[0, T]}^{4} .
$$

Then, in (35), we have

$$
\mathbb{E}\left[\left|I_{n}^{\prime \prime}(\tau)\right|^{p / 2}\right]=\mathbb{E}_{\Omega_{2}}\left[\left\|I_{n}^{\prime \prime}(\tau)\right\|_{L^{p / 2}\left(\Omega_{1}\right)}^{p / 2}\right] \leq \mathbb{E}_{\Omega_{2}}\left[\left\|I_{n}^{\prime \prime}(\tau)\right\|_{L^{2}\left(\Omega_{1}\right)}^{p / 2}\right] \lesssim \tau^{-p} \mathbb{E}_{\Omega_{2}}\left\|\phi_{n}\left(\cdot, \omega_{2}\right)\right\|_{L^{2}[0, T]}^{p}
$$

for $p \in[2,4]$. The rest follows as before.

Now, we discuss the continuity of the stochastic convolution. In the remaining of this section, we show that the stochastic convolution $\Phi(t)$ defined in (10) belongs to $C\left([0, T] ; \hat{b}_{p, \infty}^{s}(\mathbb{T})\right)$ almost surely. With $\beta_{n}=\beta_{n}^{(r)}+i \beta_{n}^{(i)}$, we have

$$
\Phi(t)=\frac{1}{\sqrt{2}} \sum_{n \neq 0} \int_{0}^{t} S(t-r) \phi_{n}(r) e_{n} d \beta_{n}^{(r)}(r)+i \frac{1}{\sqrt{2}} \sum_{n \neq 0} \int_{0}^{t} S(t-r) \phi_{n}(r) e_{n} d \beta_{n}^{(i)}(r),
$$

since $\phi e_{0}=0$ and $\phi e_{n}=\phi_{n} e_{n}, n \neq 0$. In the following, we only show the continuity of the first stochastic convolution in (37), which we denote by $\Phi^{(r)}(t)$. Also, let $W^{(r)}(t)=\frac{1}{\sqrt{2}} \sum_{n} \beta_{n}^{(r)}(t) e_{n}$. As in [Da Prato 2004], we use the factorization method based on the elementary identity

$$
\int_{r}^{t}\left(t-t^{\prime}\right)^{\alpha-1}\left(t^{\prime}-r\right)^{-\alpha} d t^{\prime}=\frac{\pi}{\sin \pi \alpha}
$$

with $\alpha \in(0,1)$ for $0 \leq r \leq t^{\prime} \leq t$. Using (38), we can write the first term in (37) as

$$
\Phi^{(r)}(t)=\frac{\sin \pi \alpha}{\pi} \int_{0}^{t} S\left(t-t^{\prime}\right)\left(t-t^{\prime}\right)^{\alpha-1} Y\left(t^{\prime}\right) d t^{\prime}
$$

where

$$
Y\left(t^{\prime}\right)=\int_{0}^{t^{\prime}} S\left(t^{\prime}-r\right)\left(t^{\prime}-r\right)^{-\alpha} \phi(r) d W^{(r)}(r)
$$

First, we present a lemma that provides a criterion for the continuity of (39) in terms of the $L^{2 m}$ integrability of $Y\left(t^{\prime}\right)$.

Lemma 4.3 [Da Prato 2004, Lemma 2.7]. Let $T>0, \alpha \in(0,1)$, and $m>\frac{1}{2 \alpha}$. For $f \in L^{2 m}\left([0, T] ; \hat{b}_{p, \infty}^{s}(\mathbb{T})\right)$, let

$$
F(t)=\int_{0}^{t} S\left(t-t^{\prime}\right)\left(t-t^{\prime}\right)^{\alpha-1} f\left(t^{\prime}\right) d t^{\prime}, \quad 0 \leq t \leq T
$$


Then, $F \in C\left([0, T] ; \hat{b}_{p, \infty}^{s}(\mathbb{T})\right)$. Moreover, there exists $C=C(m, T)$ such that

$$
\|F(t)\|_{\hat{b}_{p, \infty}^{s}} \leq C\|f\|_{L^{2 m}\left([0, T] ; \hat{b}_{p, \infty}^{s}\right)}, \quad 0 \leq t \leq T .
$$

Remark 4.4. Although Da Prato states his Lemma 2.7 for a Hilbert space $H$, his proof makes no use of the Hilbert space structure of $H$. Thus the same result holds for $\hat{b}_{p, \infty}^{s}(\mathbb{T})$ as well.

In view of Lemma 4.3 , it suffices to show that $Y\left(t^{\prime}\right) \in L^{2 m}\left([0, T] ; \hat{b}_{p, \infty}^{s}(\mathbb{T})\right)$ a.s.

Proposition 4.5. Let $T>0, m \geq 2, s=-\frac{1}{2}+$, and $p=2+$ such that $s p<-1$. Let $\phi$ be as in (3). Then, the stochastic convolution $\Phi^{(r)}(t)$ is continuous from $[0, T]$ into $\hat{b}_{p, \infty}^{s}$ almost surely. Moreover, there exists

$$
\mathbb{E}\left(\sup _{t \in[0, T]}\left\|\Phi^{(r)}(t)\right\|_{\hat{b}_{p, \infty}^{s}}^{2 m}\right) \leq C(m, T, s, p)<\infty .
$$

Proof. Let $\alpha \in\left(\frac{1}{2 m}, \frac{1}{2}\right)$ and $Y$ as in (40). First, note that $Y$ is real-valued since $\phi_{-n}(s) e_{-n}=\overline{\phi_{n}(s) e_{n}}$ and $\beta_{-n}^{(r)}=\beta_{n}^{(r)}$. Note that $\left\{\beta_{n}^{(r)}\right\}_{n \neq 0}$ and $\phi$ are independent since $\phi$ depends only on $\beta_{0}$. Thus, we can $\operatorname{regard} \beta_{n}^{(r)}$ and $\phi$ as $\beta_{n}^{(r)}(\omega)=\beta_{n}^{(r)}\left(\omega_{1}\right)$ and $\phi(\omega)=\phi\left(\omega_{2}\right)$, where $\omega=\left(\omega_{1}, \omega_{2}\right) \in \Omega_{1} \times \Omega_{2}=\Omega$. Then, for each fixed $\omega_{2}$ and $t^{\prime} \in[0, t], \widehat{Y\left(t^{\prime}\right)}(n)$ is a Gaussian random variable on $\Omega_{1}$ with $\operatorname{Var}_{\Omega_{1}}\left(\widehat{Y\left(t^{\prime}\right)}(n)\right)=$ $\mathbb{E}_{\Omega_{1}}\left[\left|\widehat{Y\left(t^{\prime}\right)}(n)\right|^{2}\right]$.

Let $G_{n}\left(r, \omega_{2}\right)=\left(t^{\prime}-r\right)^{-\alpha} e^{i\left(t^{\prime}-r\right) n^{3}} \phi_{n}\left(r, \omega_{2}\right)$. Note that $\left|G_{n}\left(r, \omega_{2}\right)\right|=\left(t^{\prime}-r\right)^{-\alpha}$ for $0<r<t^{\prime}$ and $n \neq 0$. By the Ito isometry, we have

$$
\mathbb{E}_{\Omega_{1}}\left[\left|\widehat{Y\left(t^{\prime}\right)}(n)\right|^{2}\right]=\frac{1}{2} \mathbb{E}_{\Omega_{1}}\left[\left|\int_{0}^{t^{\prime}} G_{n}\left(r, \omega_{2}\right) d \beta\left(r, \omega_{1}\right)\right|^{2}\right]=\frac{1}{2} \int_{0}^{t^{\prime}}\left|G_{n}\left(r, \omega_{2}\right)\right|^{2} d r \sim \int_{0}^{t^{\prime}}\left(t^{\prime}-r\right)^{-2 \alpha} d r .
$$

By the Minkowski integral inequality (with $p=2+<2 m$ ) after replacing $\sup _{j}$ by $\sum_{j}$, we have

$$
\begin{aligned}
\mathbb{E}_{\Omega_{1}}\left(\left\|Y\left(t^{\prime}, \cdot, \omega_{2}\right)\right\|_{\hat{b}_{p, \infty}^{s}}^{2 m}\right) & =\mathbb{E}_{\Omega_{1}}\left[\left(\sup _{j} \sum_{|n| \sim 2^{j}}\langle n\rangle^{s p}\left|\widehat{Y\left(t^{\prime}\right)}(n)\right|^{p}\right)^{2 m / p}\right] \\
& \lesssim\left(\sum_{j=0}^{\infty} \sum_{|n| \sim 2^{j}} 2^{j s p}\left(\mathbb{E}_{\Omega_{1}}\left[\left|\widehat{Y\left(t^{\prime}\right)}(n)\right|^{2 m}\right]\right)^{p / 2 m}\right)^{2 m / p} \\
& \sim\left(\sum_{j=0}^{\infty} 2^{j(s p+1)}\right)^{2 m / p}\left(\int_{0}^{t^{\prime}}\left(t^{\prime}-r\right)^{-2 \alpha} d r\right)^{m} \lesssim\left(\frac{\left(t^{\prime}\right)^{1-2 \alpha}}{1-2 \alpha}\right)^{m},
\end{aligned}
$$

since $s p<-1$. Therefore

$$
\begin{aligned}
\int_{0}^{T} \mathbb{E}( & \left.\left\|Y\left(t^{\prime}\right)\right\|_{\hat{b}_{p, \infty}^{s}}^{2 m}\right) d t^{\prime}=\int_{0}^{T} \mathbb{E}_{\Omega_{2}} \mathbb{E}_{\Omega_{1}}\left(\left\|Y\left(t^{\prime}\right)\right\|_{\hat{b}_{p, \infty}^{s}}^{2 m}\right) d t^{\prime} \\
& \lesssim \int_{0}^{T}\left(\frac{\left(t^{\prime}\right)^{1-2 \alpha}}{1-2 \alpha}\right)^{m} d t^{\prime} \lesssim T^{(1-2 \alpha) m+1}<C(m, T, s, p)<\infty .
\end{aligned}
$$

In particular, it follows that $Y(\cdot, \omega) \in L^{2 m}\left([0, T] ; \hat{b}_{p, \infty}^{s}\right)$ almost surely. Then, the desired result follows from Lemma 4.3. 


\section{Nonlinear estimate on the second iteration}

Now, we present the crucial nonlinear analysis. First, we briefly go over Bourgain's argument [1997]. By writing the integral equation, the deterministic $\mathrm{KdV}(5)$ is equivalent to

$$
u(t)=S(t) u_{0}-\frac{1}{2} \mathcal{N}(u, u)(t),
$$

where $\mathcal{N}(\cdot, \cdot)$ is given by

$$
\mathcal{N}\left(u_{1}, u_{2}\right)(t):=\int_{0}^{t} S\left(t-t^{\prime}\right) \partial_{x}\left(u_{1} u_{2}\right)\left(t^{\prime}\right) d t^{\prime}
$$

In the following, we assume that the initial condition $u_{0}$ has mean 0 , which implies that $u(t)$ has spatial mean 0 for each $t \in \mathbb{R}$. We use $(n, \tau),\left(n_{1}, \tau_{1}\right)$, and $\left(n_{2}, \tau_{2}\right)$ to denote the Fourier variables for $u u$, the first factor, and the second factor $u$ of $u u$ in $\mathcal{N}(u, u)$, respectively, thus we have $n=n_{1}+n_{2}$ and $\tau=\tau_{1}+\tau_{2}$. By the mean-zero assumption on $u$ and since we have $\partial_{x}(u u)$ in the definition of $\mathcal{N}(u, u)$, we assume $n, n_{1}, n_{2} \neq 0$. We also use the following notation:

$$
\sigma_{0}:=\left\langle\tau-n^{3}\right\rangle \text { and } \sigma_{j}:=\left\langle\tau_{j}-n_{j}^{3}\right\rangle .
$$

One of the main ingredients is the observation due to Bourgain [1993]:

$$
n^{3}-n_{1}^{3}-n_{2}^{3}=3 n n_{1} n_{2} \quad \text { for } n=n_{1}+n_{2},
$$

which in turn implies that

$$
\operatorname{MAX}:=\max \left(\sigma_{0}, \sigma_{1}, \sigma_{2}\right) \gtrsim\left\langle n n_{1} n_{2}\right\rangle
$$

Now, define

$$
A_{j}=\left\{\left(n, n_{1}, n_{2}, \tau, \tau_{1}, \tau_{2}\right) \in \mathbb{Z}^{3} \times \mathbb{R}^{3}: \sigma_{j}=\operatorname{MAX}\right\},
$$

and let $\mathcal{N}_{j}(u, u)$ denote the contribution of $\mathcal{N}(u, u)$ on $A_{j}$. By the standard bilinear estimate as in [Bourgain 1993; Kenig et al. 1996], we have

$$
\left\|\mathcal{N}_{0}(u, u)\right\|_{-\frac{1}{2}+\delta, \frac{1}{2}-\delta} \leq o(1)\|u\|_{-\frac{1}{2}-\delta, \frac{1}{2}-\delta}^{2},
$$

where $o(1)=T^{\theta}$ with some $\theta>0$ by considering the estimate on a short time interval $[-T, T]$ (for example, Lemma 3.4). See (2.17), (2.26), and (2.68) in [Bourgain 1997]. Here, we abuse the notation and use $\|\cdot\|_{s, b}=\|\cdot\|_{X^{s, b}}$ to denote the local-in-time version as well. Note that the temporal regularity $b$ equals $\frac{1}{2}-\delta<\frac{1}{2}$. This allows us to improve the spatial regularity by $2 \delta$. Clearly, we cannot expect to do the same for $\mathcal{N}_{1}(u, u)$. (By symmetry, we do not consider $\mathcal{N}_{2}(u, u)$ in the following.) The bilinear estimate (7) is known to fail for any $s \in \mathbb{R}$ if $b<\frac{1}{2}$ due to the contribution from $\mathcal{N}_{1}(u, u)$ [Kenig et al. 1996]. Following the notation in [Bourgain 1997], let

$$
I_{s, b}=\left\|\mathcal{N}_{1}(u, u)\right\|_{X^{s, b}} \quad \text { and } \quad \alpha:=\frac{1}{2}-\delta<\frac{1}{2} .
$$

Then, by Lemma 3.2 and duality with $\|d(n, \tau)\|_{L_{n, \tau}^{2}} \leq 1$, we have

$$
I_{-\alpha, 1-\alpha}=\left\|\mathcal{N}_{1}(u, u)\right\|_{-\alpha, 1-\alpha} \lesssim \sum_{\substack{n, n_{1} \\ n=n_{1}+n_{2}}} \int_{\tau=\tau_{1}+\tau_{2}} d \tau d \tau_{1} \frac{\langle n\rangle^{1-\alpha} d(n, \tau)}{\sigma_{0}^{\alpha}} \hat{u}\left(n_{1}, \tau_{1}\right) \frac{\left\langle n_{2}\right\rangle^{1-\alpha} c\left(n_{2}, \tau_{2}\right)}{\sigma_{2}^{\alpha}}
$$


where

$$
c\left(n_{2}, \tau_{2}\right)=\left\langle n_{2}\right\rangle^{-(1-\alpha)} \sigma_{2}^{\alpha} \hat{u}\left(n_{2}, \tau_{2}\right) \quad \text { so that }\|c\|_{L_{n, \tau}^{2}}=\|u\|_{-(1-\alpha), \alpha}=\|u\|_{-\frac{1}{2}-\delta, \frac{1}{2}-\delta} .
$$

The main idea here is to consider the second iteration, that is, substitute (41) for $\hat{u}\left(n_{1}, \tau_{1}\right)$ in (48), thus leading to a trilinear expression. Since $\sigma_{1}=\operatorname{MAX} \gtrsim\left\langle n n_{1} n_{2}\right\rangle \gg 1$ on $A_{1}$, we can assume that

$$
\hat{u}\left(n_{1}, \tau_{1}\right)=(\mathcal{N}(u, u))^{\wedge}\left(n_{1}, \tau_{1}\right) \sim \frac{\left|n_{1}\right|}{\sigma_{1}} \sum_{n_{1}=n_{3}+n_{4}} \int_{\tau_{1}=\tau_{3}+\tau_{4}} \hat{u}\left(n_{3}, \tau_{3}\right) \hat{u}\left(n_{4}, \tau_{4}\right) d \tau_{4} .
$$

Note that $\hat{u}\left(n_{1}, \tau_{1}\right)$ cannot come from $S(t) u_{0}$ of (41) since we have $\sigma_{1} \sim 1$ for the linear part. Moreover, by the standard computation [Bourgain 1993], we have

$$
\begin{aligned}
\mathcal{N}(u, u)(x, t)= & -i \sum_{k=1}^{\infty} \frac{i^{k} t^{k}}{k !} \sum_{n \neq 0} e^{i\left(n x+n^{3} t\right)} \int \eta\left(\lambda-n^{3}\right) \widehat{\partial_{x} u^{2}}(n, \lambda) d \lambda \\
& +i \sum_{n \neq 0} e^{i n x} \int \frac{(1-\eta)\left(\tau-n^{3}\right)}{\tau-n^{3}} \widehat{\partial_{x} u^{2}}(n, \tau) e^{i \tau t} d \tau \\
& +i \sum_{n \neq 0} e^{i\left(n x+n^{3} t\right)} \int \frac{(1-\eta)\left(\lambda-n^{3}\right)}{\lambda-n^{3}} \widehat{\partial_{x} u^{2}}(n, \lambda) d \lambda \\
= & : M_{1}(u, u)(x, t)+M_{2}(u, u)(x, t)+M_{3}(u, u)(x, t) .
\end{aligned}
$$

Note that $\left(M_{1}(u, u)\right)^{\wedge}\left(n_{1}, \tau_{1}\right)$ and $\left(M_{3}(u, u)\right)^{\wedge}\left(n_{1}, \tau_{1}\right)$ are distributions supported on $\left\{\tau_{1}-n_{1}^{3}=0\right\}$, so $\sigma_{1} \sim 1$. Hence, the only contribution for the second iteration on $A_{1}$ comes from $\mu_{2}(u, u)$ whose Fourier transform is given in (50). This shows the validity of the assumption (50).

The $\sigma_{1}$ appearing in the denominator allows us to cancel $\langle n\rangle^{1-\alpha}$ and $\left\langle n_{2}\right\rangle^{1-\alpha}$ in the numerator in (48). Then, $I_{-\alpha, 1-\alpha}$ can be estimated by

$$
\lesssim \sum_{\substack{n=n_{1}+n_{2} \\ n_{1}=n_{3}+n_{4}}} \int_{\substack{\tau=\tau_{1}+\tau_{2} \\ \tau_{1}=\tau_{3}+\tau_{4}}} \frac{\langle n\rangle^{1-\alpha} d(n, \tau)}{\sigma_{0}^{\alpha}} \frac{\left|n_{1}\right|}{\sigma_{1}} \hat{u}\left(n_{3}, \tau_{3}\right) \hat{u}\left(n_{4}, \tau_{4}\right) \frac{\left\langle n_{2}\right\rangle^{1-\alpha} c\left(n_{2}, \tau_{2}\right)}{\sigma_{2}^{\alpha}} .
$$

Bourgain then divided the argument into several cases, depending on the sizes of $\sigma_{0}, \ldots, \sigma_{4}$. Here, the key algebraic relation is

$$
n^{3}-n_{2}^{3}-n_{3}^{3}-n_{4}^{3}=3\left(n_{2}+n_{3}\right)\left(n_{3}+n_{4}\right)\left(n_{4}+n_{2}\right) \quad \text { with } n=n_{2}+n_{3}+n_{4} .
$$

Then, Bourgain proved [1997, (2.69)]

$$
I_{-\alpha, 1-\alpha} \leq o(1)\|u\|_{-(1-\alpha), \alpha} I_{-\alpha, 1-\alpha}+o(1)\|u\|_{-(1-\alpha), \alpha}^{3}+o(1)\|u\|_{-(1-\alpha), \alpha},
$$

assuming the a priori estimate (8): $|\hat{u}(n, t)|<C$ for all $n \in \mathbb{Z}, t \in \mathbb{R}$. Indeed, the estimates involving the first two terms on the right-hand side of (54) were obtained without (8), and only the last term in (54) required (8) [Bourgain 1997, "Estimation of (2.62)"], which was then used to deduce

$$
\|\hat{u}(n, \cdot)\|_{L_{\tau}^{2}}<C
$$


The a priori estimate (8) is derived via the isospectral property of the $\mathrm{KdV}$ flow and is false for a general function in $X^{-(1-\alpha), \alpha}$. (It is here that the smallness of the total variation $\|\mu\|$ is used.)

Our goal is to carry out a similar analysis for SKdV (2) on the second iteration without the a priori estimates (8) and (55) coming from the complete integrability of $\mathrm{KdV}$. We achieve this goal by considering the estimate in

$$
X_{p, 2}^{-\alpha, \alpha}=X_{p, 2}^{-\frac{1}{2}+\delta, \frac{1}{2}-\delta}
$$

where $p=2+$ and $\frac{p-2}{4 p}<\delta<\frac{p-2}{2 p}$. By (15) and (17) (recall $-\alpha=-\frac{1}{2}+\delta$ and $-(1-\alpha)=-\frac{1}{2}-\delta$ ), we have

$$
\|u\|_{X_{p, 2}^{-\alpha, \alpha}} \leq\|u\|_{X^{-\alpha, \alpha}} \quad \text { and } \quad\|u\|_{X^{-(1-\alpha), \alpha}} \lesssim\|u\|_{X_{p, 2}^{-\alpha, \alpha}}
$$

Then, it follows from (46) and (56) that

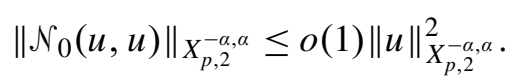

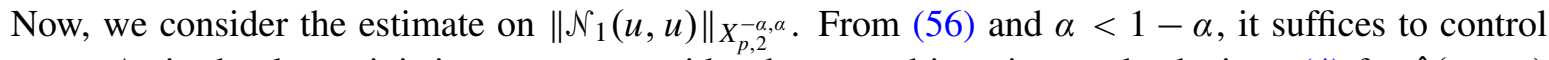
$I_{-\alpha, 1-\alpha}$. As in the deterministic case, we consider the second iteration, and substitute (4) for $\hat{u}\left(n_{1}, \tau_{1}\right)$ in (48). As before, there is no contribution from $S(t) u_{0}$, or $M_{1}(u, u), M_{3}(u, u)$ defined in (51). There are two contributions:

(i) $\mathcal{N}_{1}\left(M_{2}(u, u), u\right)$ from the deterministic nonlinear part: In this case, we can use the estimates from [Bourgain 1997] except when the a priori bound (8) was assumed; that is, we need to estimate the contribution from [Bourgain 1997, (2.62)]:

$$
R_{\alpha}:=\sum_{n} \int_{\tau=\tau_{2}+\tau_{3}+\tau_{4}} \chi_{B} \frac{d(n, \tau)}{\langle n\rangle^{1+\alpha} \sigma_{0}^{\alpha}} \hat{u}\left(-n, \tau_{2}\right) \hat{u}\left(n, \tau_{3}\right) \hat{u}\left(n, \tau_{4}\right) d \tau_{2} d \tau_{3} d \tau_{4},
$$

where $\|d(n, \tau)\|_{L_{n, \tau}^{2}} \leq 1$ and $B=\left\{\sigma_{0}, \sigma_{2}, \sigma_{3}, \sigma_{4}<|n|^{\gamma}\right\}$ with some small parameter $\gamma>0$. Note that this corresponds to the case $n_{2}=-n$ and $n_{3}=n_{4}=n$ in (52) after some reduction. In our

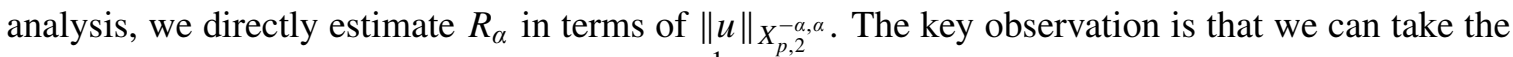
spatial regularity $s=-\alpha$ to be greater than $-\frac{1}{2}$ by choosing $p>2$.

(ii) $\mathcal{N}_{1}(\Phi, u)$ from the stochastic convolution $\Phi$ in (10): In view of (56), we estimate

$$
\mathbb{E}\left[\left\|\mathcal{N}_{1}(\eta \Phi, u)\right\|_{\left.X^{-\alpha, 1-\alpha}\right]}\right.
$$

via the stochastic analysis from Section 4.

Remark 5.1. In fact, we do not need to take an expectation in (59) since we establish local wellposedness pathwise in $\omega$, that is, for almost every fixed $\omega$. Nonetheless, we estimate (59) with the expectation since it shows how $F_{1}^{N}$ and $F_{2}^{N}$ defined in (70) arise along with their estimates.

Estimate on (i). In [Bourgain 1997], the parameter $\gamma=\gamma(\alpha)$, subject to the conditions (2.43) and (2.60) therein, played a certain role in estimating $R_{\alpha}$ along with the a priori bound (8). However, it plays no role in our analysis. By the Cauchy-Schwarz and Young's inequalities, we have

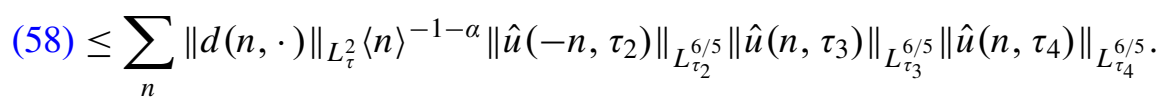


By the Hölder inequality (with appropriate \pm signs) and the fact that $-1-\alpha<-3 \alpha$,

$$
(58) \leq \sum_{n}\|d(n, \cdot)\|_{L_{\tau}^{2}} \prod_{j=2}^{4}\langle n\rangle^{-\alpha-}\left\|\sigma_{j}^{-\alpha}\right\|_{L_{\tau_{j}}^{3}}\left\|\sigma_{j}^{\alpha} \hat{u}\left( \pm n, \tau_{j}\right)\right\|_{L_{\tau_{j}}^{2}} \leq\|d(\cdot, \cdot)\|_{L_{n, \tau}^{2}}\|u\|_{X_{6,2}^{-\alpha, \alpha}}^{3} \leq\|u\|_{X_{p, 2}^{-\alpha, \alpha}}^{3},
$$

where the last two inequalities follow by choosing $\alpha>\frac{1}{3}$ and $p=2+<6$.

Estimate on (ii). We use the notation from the proof of Proposition 4.1. It follows from (28) and $\eta(t) \Phi(\cdot, t)=S(t) g(\cdot, t)$ that

$$
(\eta \Phi)^{\wedge}\left(n_{1}, \tau_{1}\right)=\hat{g}\left(n_{1}, \tau_{1}-n_{1}^{3}\right)=\frac{1}{\sqrt{2}} \mathbf{I}_{n_{1}}^{(1)}\left(\tau_{1}-n_{1}^{3}\right)+\frac{1}{\sqrt{2}} \mathrm{I}_{n_{1}}^{(2)}\left(\tau_{1}-n_{1}^{3}\right) .
$$

Recall that $\sigma_{1}=\left\langle\tau_{1}-n_{1}^{3}\right\rangle \gtrsim\left\langle n n_{1} n_{2}\right\rangle$. Also, recall from the proof of Proposition 4.1 that $\left|\phi_{n_{1}}(r)\right|=\chi_{[0, T]}(r)$ is independent of $\omega$.

- Contribution from $\mathrm{I}_{n_{1}}^{(1)}\left(\tau_{1}-n_{1}^{3}\right)$ : From (48) with (27), (28), and (29), we estimate (59) by

$$
\text { (59) } \lesssim \mathbb{E}\left[\sum_{\substack{n, n_{1} \\ n=n_{1}+n_{2}}} \int_{\tau=\tau_{1}+\tau_{2}} d \tau d \tau_{1} \frac{\langle n\rangle^{1-\alpha} d(n, \tau)}{\sigma_{0}^{\alpha}} \frac{1}{\sigma_{1}^{2}} \int_{0}^{T}\left|\phi_{n_{1}}(r)\right| d \beta_{n_{1}}(r) \frac{\left\langle n_{2}\right\rangle^{1-\alpha} c\left(n_{2}, \tau_{2}\right)}{\sigma_{2}^{\alpha}}\right] .
$$

By the Cauchy-Schwarz inequality in $\omega$ and the Ito isometry,

$$
(59) \lesssim \sum_{\substack{n, n_{1} \\ n=n_{1}+n_{2}}} \int_{\tau=\tau_{1}+\tau_{2}} d \tau d \tau_{1} \frac{d(n, \tau)}{\sigma_{0}^{\alpha}} \frac{\left\|\phi_{n_{1}}\right\|_{L^{2}[0, T]}}{\sigma_{1}^{(3 / 2)-\delta}\left\langle n_{1}\right\rangle^{(1 / 2)+\delta}} \frac{\left\|c\left(n_{2}, \tau_{2}\right)\right\|_{L^{2}(\Omega)}}{\sigma_{2}^{\alpha}} .
$$

By the $L_{x, t}^{4}, L_{x, t}^{2}, L_{x, t}^{4}$-Hölder inequality along with Lemma 3.3, (16), (18), (49), and (56), this leads to

$$
\begin{aligned}
(59) & \lesssim T^{\theta}\|d\|_{L_{n, \tau}^{2}}\|\phi\|_{L^{2}\left([0, T] ; H^{-\frac{1}{2}-\delta}\right)}\|c\|_{L^{2}\left(\Omega ; L_{n, \tau}^{2}\right)} \leq T^{\theta}\|\phi\|_{L^{p}\left([0, T] ; \hat{b}_{p, \infty}^{-\alpha}\right)}\|u\|_{L^{2}\left(\Omega ; X^{-(1-\alpha), \alpha}\right)} \\
& \lesssim T^{\theta}\|\phi\|_{L^{p}\left([0, T] ; \hat{b}_{p, \infty}^{-\alpha}\right)}\|u\|_{L^{2}\left(\Omega ; X_{p, 2}^{-\alpha, \alpha}\right)} .
\end{aligned}
$$

Remark 5.2. Strictly speaking, we need to take the supremum over $\left\{\|d\|_{L_{n, \tau}^{2}}=1\right\}$ inside the expectation in (60). However, we do not worry about this issue to simplify the presentation, since we have

$$
\begin{aligned}
(59) & \leq\left\|\mathcal{N}_{1}(\eta \Phi, u)\right\|_{L^{2}\left(\Omega ; X^{-\alpha, 1-\alpha}\right)} \\
& \leq\left(\sum_{n} \int \frac{\langle n\rangle^{2-2 \alpha}}{\sigma_{0}^{2 \alpha}} \mathbb{E}\left[\left|\int_{0}^{T}\right| \phi_{n_{1}}(r)\left|\sum_{n=n_{1}+n_{2}} \int_{\tau=\tau_{1}+\tau_{2}} \frac{\left\langle n_{2}\right\rangle^{1-\alpha} c\left(n_{2}, \tau_{2}\right)}{\sigma_{1}^{2} \sigma_{2}^{\alpha}} d \tau_{1} d \beta_{n_{1}}(r)\right|^{2}\right] d \tau\right)^{1 / 2} \\
& =\sup _{\|d\|_{L_{n, \tau}^{2}}=1}(61)
\end{aligned}
$$

by the Ito isometry. Also, recall that we have $\mathrm{I}_{n_{1}}^{(1)}\left(\tau_{1}-n_{1}^{3}\right)=\int_{0}^{T} G_{n_{1}}^{(1)}\left(r, \tau_{1}-n_{1}^{3}\right) d \beta_{n_{1}}(r)$ where $G_{n}^{(1)}(r, \tau)$ is defined in (27). Hence, strictly speaking, we should replace $G_{n_{1}}^{(1)}\left(r, \tau_{1}-n_{1}^{3}\right)$ by $\sigma_{1}^{-2}\left|\phi_{n_{1}}(r)\right|$ in (60) only after the application of the Ito isometry. Once again, we do not worry about this issue to simplify the presentation. The same remark applies to the following as well. 
- Contribution from $\mathrm{I}_{n_{1}}^{(2)}\left(\tau_{1}-n_{1}^{3}\right)$ : Suppose $\max \left(\sigma_{0}, \sigma_{2}\right) \gtrsim\left\langle n n_{1} n_{2}\right\rangle^{1 / 100}$; say $\sigma_{0} \geq\left\langle n n_{1} n_{2}\right\rangle^{1 / 100}$. Then

$$
\begin{aligned}
(59) & \lesssim \mathbb{E}\left[\sum_{\substack{n, n_{1} \\
n=n_{1}+n_{2}}} \int_{\tau=\tau_{1}+\tau_{2}} d \tau d \tau_{1} \frac{\langle n\rangle^{1-\alpha} d(n, \tau)}{\sigma_{0}^{\alpha}} \frac{1}{\sigma_{1}} \int_{0}^{T}\left|\phi_{n_{1}}(r)\right| d \beta_{n_{1}}(r) \frac{\left\langle n_{2}\right\rangle^{1-\alpha} c\left(n_{2}, \tau_{2}\right)}{\sigma_{2}^{\alpha}}\right] \\
& \lesssim \sum_{\substack{n, n_{1} \\
n=n_{1}+n_{2}}} \int_{\tau=\tau_{1}+\tau_{2}} d \tau d \tau_{1} \frac{d(n, \tau)}{\sigma_{0}^{\alpha-200 \delta}} \frac{\left\|\phi_{n_{1}}\right\|_{L^{2}[0, T]}}{\sigma_{1}^{(1 / 2)+\delta}\left\langle n_{1}\right\rangle^{(1 / 2)+\delta}} \frac{\left\|c\left(n_{2}, \tau_{2}\right)\right\|_{L^{2}(\Omega)}}{\sigma_{2}^{\alpha}} .
\end{aligned}
$$

Then we can conclude this case as before by the $L_{x, t}^{4}, L_{x, t}^{2}, L_{x, t}^{4}$-Hölder inequality as long as $\alpha-200 \delta>\frac{1}{3}$, which can be guaranteed by taking $\delta>0$ sufficiently small, or equivalently, taking $p>2$ sufficiently close to 2 .

Now assume instead $\max \left(\sigma_{0}, \sigma_{2}\right) \ll\left\langle n n_{1} n_{2}\right\rangle^{1 / 100}$. We invoke a result contained in [Colliander et al. 2003, (7.50) and Lemma 7.4]. The conclusion there is stated with -1 as the exponent of $\left\langle\tau-n^{3}\right\rangle$, instead of $-\frac{3}{4}$; but by examining the proof, one sees that it will work with any exponent more negative than $-\left(\frac{2}{3}+\frac{1}{100}\right)$.

Lemma 5.3. For $\Omega(n)=\left\{\eta \in \mathbb{R}: \eta=-3 n n_{1} n_{2}+o\left(\left\langle n n_{1} n_{2}\right\rangle^{1 / 100}\right)\right.$ for some $n_{1} \in \mathbb{Z}$ with $\left.n=n_{1}+n_{2}\right\}$,

$$
\int\left\langle\tau-n^{3}\right\rangle^{-3 / 4} \chi_{\Omega(n)}\left(\tau-n^{3}\right) d \tau \lesssim 1
$$

We have

$$
(59) \lesssim \mathbb{E}\left[\sum_{\substack{n, n_{1} \\ n=n_{1}+n_{2}}} \int_{\tau=\tau_{1}+\tau_{2}} d \tau d \tau_{1} \frac{\langle n\rangle^{1-\alpha} d(n, \tau)}{\sigma_{0}^{\alpha}} \frac{\chi_{\Omega\left(n_{1}\right)}\left(\tau_{1}-n_{1}^{3}\right)}{\sigma_{1}} \int_{0}^{T}\left|\phi_{n_{1}}(r)\right| d \beta_{n_{1}}(r) \frac{\left\langle n_{2}\right\rangle^{1-\alpha} c\left(n_{2}, \tau_{2}\right)}{\sigma_{2}^{\alpha}}\right]
$$

By the Cauchy-Schwarz inequality and the Ito isometry, this yields

$$
(59) \lesssim \sum_{\substack{n, n_{1} \\ n=n_{1}+n_{2}}} \int_{\tau=\tau_{1}+\tau_{2}} d \tau d \tau_{1} \frac{d(n, \tau)}{\sigma_{0}^{\alpha}} \frac{\chi_{\Omega\left(n_{1}\right)}\left(\tau_{1}-n_{1}^{3}\right)\left\|\phi_{n_{1}}\right\|_{L^{2}[0, T]}}{\sigma_{1}^{(1 / 2)-\delta}\left\langle n_{1}\right\rangle^{(1 / 2)+\delta}} \frac{\left\|c\left(n_{2}, \tau_{2}\right)\right\|_{L^{2}(\Omega)}}{\sigma_{2}^{\alpha}}
$$

By the $L_{x, t}^{4}, L_{x, t}^{2}, L_{x, t}^{4}$-Hölder inequality along with Lemma 3.3, Lemma 5.3, and Equations (16), (18), (49), and (56), we get

$$
\begin{aligned}
(59) & \lesssim T^{\theta}\|d\|_{L_{n, \tau}^{2}}\left\|\left\langle n_{1}\right\rangle^{-\frac{1}{2}-\delta}\right\| \phi_{n_{1}}\left\|_{L^{2}[0, T]}\right\| \chi \Omega\left(n_{1}\right) \\
& \left.\leq T^{\theta}\|\phi\|_{L^{2}\left([0, T] ; H^{-\frac{1}{2}-\delta}\right)}\|u\|_{L^{2}\left(\Omega ; X^{-(1-\alpha), \alpha}\right)} \lesssim T^{\theta}\right) \sigma_{1}^{-\frac{1}{2}+\delta}\|\phi\|_{L^{p}\left([0, T] ; \hat{b}_{p, \infty}^{-\alpha}\right)}\|u\|_{L^{2}\left(\Omega ; X_{p, 2}^{-\alpha, \alpha}\right)}\|c\|_{L^{2}\left(\Omega ; L_{n, \tau}^{2}\right)} .
\end{aligned}
$$

Proof of Theorem 1. Fix a mean-zero $u_{0} \in \hat{b}_{p, \infty}^{-\alpha^{\prime}}(\mathbb{T})$ and $\phi$ as in (3), where $\alpha^{\prime}=\frac{1}{2}-\delta-$ with $\frac{p-2}{4 p}<\delta<\frac{p-2}{2 p}$ such that $\left(-\alpha^{\prime}\right) p<-1$. Consider sequences of initial data $u_{0}^{N} \in L^{2}(\mathbb{T})$ and diagonal covariance operator $\phi^{N} \in H S\left(L^{2} ; L^{2}\right)$, given by

$$
u_{0}^{N}=\mathbb{P}_{\leq N} u_{0}=\sum_{|n| \leq N} \hat{u}_{0}(n) e^{i n x} \quad \text { and } \quad \phi^{N}(t, \omega):=\operatorname{diag}\left(\phi_{n}(t, \omega) ; 0<|n| \leq N\right),
$$

where $\phi_{n}$ is given in (3). Now, fix $\alpha=\frac{1}{2}-\delta>\alpha^{\prime}$ as in (47). Note that such $u_{0}^{N}$ converges to $u_{0}$ in $\mathscr{F} L^{-\alpha, p}(\mathbb{T})$, and thus in $\hat{b}_{p, \infty}^{-\alpha}(\mathbb{T})$. Also, $\phi^{N}$ converges to $\phi$ in $\mathscr{F} L^{-\frac{1}{2}-, p}(\mathbb{T})$ for each $t$ and $\omega$, and thus 
in $\hat{b}_{p, \infty}^{-\frac{1}{2}-}(\mathbb{T})$. Then, by the monotone convergence theorem, $\phi^{N}$ converges to $\phi$ in $L^{p}\left([0,1] \times \Omega ; \hat{b}_{p, \infty}^{-\frac{1}{2}-}\right)$. (Indeed, the convergence is in $L^{\infty}\left([0,1] \times \Omega ; \hat{b}_{p}^{-\frac{1}{2}-}\right)$, since we have $\left|\phi_{n}(t, \omega)\right|=1$ for all $n$, independent of $t \in \mathbb{R}$ and $\omega \in \Omega$.) Note that a slight loss of the regularity $-\alpha<-\alpha^{\prime}$ was necessary since $u_{0}^{N}$ defined in (65) does not necessarily converge to $u_{0}$ in $\hat{b}_{p, \infty}^{-\alpha^{\prime}}(\mathbb{T})$ due to the $L^{\infty}$ nature of the norm over the dyadic blocks. We can avoid such a loss of the regularity if we start with $u_{0} \in \mathscr{F} L^{s, p}(\mathbb{T})$.

Now, let $\Gamma^{N}=\Gamma_{u_{0}^{N}}^{N}$ be the map defined by

$$
\Gamma^{N} v=\Gamma_{u_{0}^{N}}^{N} v:=S(t) u_{0}^{N}-\frac{1}{2} \mathcal{N}(v, v)+\eta \Phi^{N},
$$

where $\Phi^{N}$ is the stochastic convolution defined in (10) with the covariance operator $\phi^{N}$. By the wellposedness result in [De Bouard et al. 2004], there exists a unique global solution $u^{N} \in L^{\infty}\left(\mathbb{R}^{+} ; L^{2}(\mathbb{T})\right) \cap$ $C\left(\mathbb{R}^{+} ; B_{2,1}^{0-}(\mathbb{T})\right)$ a.s. to (66) for each $N$ since $\phi^{N} \in H S\left(L^{2} ; L^{2}\right)$.

Now, we put all the estimates together. Note that all the implicit constants are independent of $N$. Also, when there is no superscript $N$, it means that $N=\infty$. From Lemma 3.1, we have

$$
\left\|S(t) u_{0}^{N}\right\|_{X_{p, 2}^{s, b, T}} \leq C_{1}\left\|u_{0}^{N}\right\|_{\hat{b}_{p, \infty}^{s}}
$$

for any $s, b \in \mathbb{R}$ with $C_{1}=C_{1}(b)$. In particular, by taking $b>\frac{1}{2}$, we see that $S(t) u_{0}$ is continuous on $[0, T]$ with values in $\hat{b}_{p, \infty}^{s}$. Also, by taking $b<\frac{1}{2}$, we gain a power of $T$. From the definition of $\mathcal{N}_{j}(\cdot, \cdot)$ and (57), we have

$$
\left\|\mathcal{N}\left(u^{N}, u^{N}\right)\right\|_{X_{p, 2}^{-\alpha, \alpha, T}} \leq C_{2} T^{\theta_{1}}\left\|u^{N}\right\|_{X_{p, 2}^{-\alpha, \alpha, T}}^{2}+2\left\|\mathcal{N}_{1}\left(u^{N}, u^{N}\right)\right\|_{X_{p, 2}^{-\alpha, \alpha, T}} .
$$

Also, from (47) and (56), we have

$$
\left\|\mathcal{N}_{1}\left(u^{N}, u^{N}\right)\right\|_{X_{p, 2}^{-\alpha, 1-\alpha, T}} \leq I_{-\alpha, 1-\alpha}^{N} .
$$

Recall that $\eta \Phi \in X_{p, 2}^{-\alpha, \alpha}$ a.s. from Proposition 4.1. Moreover, by defining $F_{1}^{N}$ and $F_{2}^{N}$ on $\mathbb{T} \times \mathbb{R} \times \Omega$ via their Fourier transforms

$$
\begin{aligned}
& \widehat{F_{1}^{N}}(n, \tau)=\langle n\rangle^{-\frac{1}{2}-\delta}\left(\sigma_{0}^{-\frac{3}{2}+\delta}+\sigma_{0}^{-\frac{1}{2}-\delta}\right) \int_{0}^{T}\left|\phi_{n}(r)\right| d \beta_{n}(r), \\
& \widehat{F_{2}^{N}}(n, \tau)=\langle n\rangle^{-\frac{1}{2}-\delta} \chi_{\Omega(n)}\left(\tau-n^{3}\right) \sigma_{0}^{-\frac{1}{2}+\delta} \int_{0}^{T}\left|\phi_{n}(r)\right| d \beta_{n}(r)
\end{aligned}
$$

for $|n| \leq N$, we have $F_{1}^{N}, F_{2}^{N} \in L^{2}\left(\Omega ; L_{x, t}^{2}\right)$ by the Ito isometry and Lemma 5.3 , which is basically shown in the estimate on (ii). See (61) and (64). Then, from (54) and the estimates on (i) and (ii), we have

$$
I_{-\alpha, 1-\alpha}^{N} \leq C_{3}\left(T^{\theta_{2}}\left\|u^{N}\right\|_{X_{p, 2}^{-\alpha, \alpha}, T} I_{-\alpha, 1-\alpha}^{N}+T^{\theta_{3}}\left\|u^{N}\right\|_{X_{p, 2}^{-\alpha, \alpha, T}}^{3}+T^{\theta_{4}} L_{\omega}^{N}\left\|u^{N}\right\|_{X_{p, 2}^{-\alpha, \alpha, T}}\right),
$$

where $L_{\omega}^{N}=L^{N}\left(F_{1}^{N}, F_{2}^{N}\right)(\omega):=\left\|F_{1}^{N}(\omega)\right\|_{L_{x, t}^{2}}+\left\|F_{2}^{N}(\omega)\right\|_{L_{x, t}^{2}}<\infty$ a.s. Moreover, $L_{\omega}^{N}$ is nondecreasing in $N$. 
For fixed $R>0$, choose $T>0$ small such that $C_{3} T^{\theta_{2}} R \leq \frac{1}{2}$. Then, from (71), we have

$$
I_{-\alpha, 1-\alpha}^{N} \leq 2 C_{3}\left(T^{\theta_{3}}\left\|u^{N}\right\|_{X_{p, 2}^{-\alpha, \alpha, T}}^{3}+T^{\theta_{4}} L_{\omega}^{N}\left\|u^{N}\right\|_{X_{p, 2}^{-\alpha, \alpha, T}}\right)
$$

for $\left\|u^{N}\right\|_{X_{p, 2}^{-\alpha, \alpha, T}} \leq R$. From (66)-(72), we have

$$
\begin{aligned}
\left\|u^{N}\right\|_{X_{p, 2}^{-\alpha, \alpha, T}}=\left\|\Gamma^{N} u^{N}\right\|_{X_{p, 2}^{-\alpha, \alpha, T}} & \leq C_{1}\left\|u_{0}^{N}\right\|_{\hat{b}_{p, \infty}^{-\alpha}}+\frac{1}{2} C_{2} T^{\theta_{1}}\left\|u^{N}\right\|_{X_{p, 2}^{-\alpha, \alpha},}^{2} \\
& +2 C_{3}\left(T^{\theta_{3}}\left\|u^{N}\right\|_{X_{p, 2}^{-\alpha, \alpha, T}}^{3}+T^{\theta_{4}} L_{\omega}^{N}\left\|u^{N}\right\|_{X_{p, 2}^{-\alpha, \alpha}, T}\right)+C_{4}\left\|\eta \Phi^{N}(\omega)\right\|_{X_{p, 2}^{-\alpha, \alpha}}
\end{aligned}
$$

and

$$
\begin{aligned}
\left\|u^{N}-u^{M}\right\|_{X_{p, 2}^{-\alpha, \alpha, T}}=\left\|\Gamma^{N} u^{N}-\Gamma^{M} u^{M}\right\|_{X_{p, 2}^{-\alpha, \alpha, T}} & \\
\leq C_{1}\left\|u_{0}^{N}-u_{0}^{M}\right\|_{\hat{b}_{p, \infty}^{-\alpha}} & +\frac{1}{2} C_{2} T^{\theta_{1}}\left(\left\|u^{N}\right\|_{X_{p, 2}^{-\alpha, \alpha}, T}+\left\|u^{M}\right\|_{X_{p, 2}^{-\alpha, \alpha}, T}\right)\left\|u^{N}-u^{M}\right\|_{X_{p, 2}^{-\alpha, \alpha},} \\
& +C_{5} T^{\theta_{3}}\left(\left\|u^{N}\right\|_{X_{p, 2}^{-\alpha, \alpha, T}}^{2}+\left\|u^{M}\right\|_{X_{p, 2}^{-\alpha, \alpha},}^{2}\right)\left\|u^{N}-u^{M}\right\|_{X_{p, 2}^{-\alpha, \alpha, T}} \\
& +2 C_{3} T^{\theta_{4}} L_{\omega}^{N}\left\|u^{N}-u^{M}\right\|_{X_{p, 2}^{-\alpha, \alpha, T}}+2 C_{3} T^{\theta_{4}} \widetilde{L}_{\omega}^{N, M}\left\|u^{M}\right\|_{X_{p, 2}^{-\alpha, \alpha},} \\
& +C_{4}\left\|\eta\left(\Phi^{N}-\Phi^{M}\right)\right\|_{X_{p, 2}^{-\alpha, \alpha}}
\end{aligned}
$$

where

$$
\widetilde{L}_{\omega}^{N, M}:=\left\|F_{1}^{N}-F_{1}^{M}\right\|_{L_{x, t}^{2}}+\left\|F_{2}^{N}-F_{2}^{M}\right\|_{L_{x, t}^{2}} .
$$

Note that in estimating the difference $\Gamma^{N} u^{N}-\Gamma^{M} u^{M}$ on $A_{1}$, one needs to consider

$$
\widetilde{I}_{-\alpha, 1-\alpha}:=\left\|\mathcal{N}_{1}\left(u^{N}, u^{N}\right)-\mathcal{N}_{1}\left(u^{M}, u^{M}\right)\right\|_{-\alpha, 1-\alpha}
$$

as in [Bourgain 1997]. We can follow the argument on pages 135-136 in that reference, except for $R_{\alpha}$ defined in (58), which yields the third term on the right-hand side of (74). As for $R_{\alpha}$, we can write

$$
\mathcal{N}(\mathcal{N}(u, u), u)-\mathcal{N}(\mathcal{N}(v, v), v)=\mathcal{N}(\mathcal{N}(u+v, u-v), u)+\mathcal{N}(\mathcal{N}(v, v), u-v)
$$

as in [Bourgain 1997, (3.4)], and then we can repeat the computation done for $R_{\alpha}$ in the estimate on (i), also yielding the third term on the right-hand side of (74).

By the definition of $u_{0}^{N}$, we have $2 C_{1}\left\|u_{0}^{N}\right\|_{\hat{b}_{p, \infty}^{-\alpha}} \leq 2 C_{1}\left\|u_{0}\right\|_{\hat{b}_{p, \infty}^{-\alpha}}+\frac{1}{2}$ for $N$ sufficiently large. And since $\phi^{N}$ converges to $\phi$ in $L^{p}\left([0,1] \times \Omega ; \hat{b}_{p, \infty}^{-\alpha+}\right)$, it follows from Corollary 4.2 and the estimate on (ii), see (61), (62), and (64), that $\mathbb{E}\left[\left\|\eta\left(\Phi^{N}-\Phi\right)\right\|_{X_{p, 2}^{-\alpha, \alpha}}\right]$ and $\mathbb{E}\left[\widetilde{L}_{\omega}^{N, \infty}\right]$ defined in (75) converge to 0. Hence, $\left\|\eta\left(\Phi^{N}-\Phi\right)\right\|_{X_{p, 2}^{-\alpha, \alpha}}+\widetilde{L}_{\omega}^{N, \infty} \rightarrow 0$ a.s. after selecting a subsequence (which we still denote with the index $N$.) Then, by Egoroff's theorem, given $\varepsilon>0$, there exists a set $\Omega_{\varepsilon}$ with $\mathbb{P}\left(\Omega_{\varepsilon}^{c}\right)<2^{-1} \varepsilon$ such that $\left\|\eta\left(\Phi^{N}-\Phi\right)\right\|_{X_{p, 2}^{-\alpha, \alpha}}+\widetilde{L}_{\omega}^{N, \infty} \rightarrow 0$ uniformly in $\Omega_{\varepsilon}$. In particular, $2 C_{4}\left\|\eta \Phi^{N}\right\|_{X_{p, 2}^{-\alpha, \alpha}} \leq 2 C_{4}\|\eta \Phi\|_{X_{p, 2}^{-\alpha, \alpha}}+\frac{1}{2}$ for large $N$ uniformly on $\Omega_{\varepsilon}$. In the following, we will work on $\Omega_{\varepsilon}$.

Now, let $R_{\omega}=2\left(C_{1}\left\|u_{0}\right\|_{\hat{b}_{p, \infty}^{-\alpha}}+C_{4}\|\eta \Phi(\omega)\|_{X_{p, 2}^{-\alpha, \alpha}}\right)+1$, and define the stopping time $T_{\omega}$ by

$$
T_{\omega}=\inf \left\{T>0: \max \left(C_{3} T^{\theta_{2}} R_{\omega}, P_{1}\left(T, R_{\omega}, \omega\right), P_{2}\left(T, R_{\omega}, \omega\right) \geq \frac{1}{2}\right\},\right.
$$

where

$$
\begin{array}{ll}
P_{1}\left(T, R_{\omega}, \omega\right)=\frac{1}{2} C_{2} T^{\theta_{1}} R_{\omega}+2 C_{3} T^{\theta_{3}}\left(R_{\omega}\right)^{2}+2 C_{3} T^{\theta_{4}} L_{\omega} & \text { from (73), } \\
P_{2}\left(T, R_{\omega}, \omega\right)=C_{2} T^{\theta_{1}} R_{\omega}+2 C_{5} T^{\theta_{3}}\left(R_{\omega}\right)^{2}+2 C_{3} T^{\theta_{4}} L_{\omega} & \text { from (74). }
\end{array}
$$


The first condition in the definition of $T_{\omega}$ guarantees (72), and hence (73) and (74), for $\left\|u^{N}\right\|_{X_{p, 2}^{-\alpha, \alpha, T}} \leq R_{\omega}$. The second condition along with (73) indeed guarantees that

$$
\left\|u^{N}\right\|_{X_{p, 2}^{-\alpha, \alpha, T}} \leq R_{\omega}
$$

for $T \leq T_{\omega}$, for the following reason. Because of the temporal regularity $b=\alpha<\frac{1}{2}$, we have $\left\|u^{N}\right\|_{X_{p, 2}^{-\alpha, \alpha}, T}=$ $\left\|\chi_{[0, T]} u^{N}\right\|_{X_{p, 2}^{-\alpha, \alpha}}$, where $\chi_{[0, T]}$ denotes the characteristic function of the time interval $[0, T]$ [Bourgain 1999]. Hence, $\left\|u^{N}\right\|_{X_{p, 2}^{-\alpha, \alpha, T}}$ is continuous in $T$ since

$$
\left|\left\|u^{N}\right\|_{X_{p, 2}^{-\alpha, \alpha, T+\delta}}-\left\|u^{N}\right\|_{X_{p, 2}^{-\alpha, \alpha, T}}\right| \leq\left\|u^{N}\right\|_{X_{p, 2}^{-\alpha, \alpha}[T, T+\delta]} \lesssim \delta^{\theta}\left\|u^{N}\right\|_{X^{0-, \frac{1}{2}}[T, T+\delta]}
$$

for sufficiently small $\delta>0$. Note that the last term in (80) is finite for small $\delta$ since the local-in-time solutions constructed in [De Bouard et al. 2004] are controlled in this norm (indeed in a stronger norm adapted to the Besov space $B_{2,1}^{0-}$.) Then, (79) follows from (73), the second condition in (78), and the continuity of the norm in $T$ since (79) clearly holds at $T=0$.

From (74) along with the third condition in (78), we have

$$
\left\|u^{N}-u^{M}\right\|_{X_{p, 2}^{-\alpha, \alpha, T_{\omega}}} \leq 2 C_{1}\left\|u_{0}^{N}-u_{0}^{M}\right\|_{\hat{b}_{p, \infty}^{-\alpha}}+4 C_{3} T^{\theta_{4}} R_{\omega} \widetilde{L}_{\omega}^{N, M}+2 C_{4}\left\|\eta\left(\Phi^{N}-\Phi^{M}\right)\right\|_{X_{p, 2}^{-\alpha, \alpha}} .
$$

The right-hand side of (81) goes to 0 as $N, M \rightarrow \infty$ since $u_{0}^{N}$ is Cauchy in $\hat{b}_{p, \infty}^{-\alpha}$ and

$$
\left\|\eta\left(\Phi^{N}-\Phi^{M}\right)\right\|_{X_{p, 2}^{-\alpha, \alpha}}+\widetilde{L}_{\omega}^{N, M} \rightarrow 0
$$

on $\Omega_{\varepsilon}$ uniformly in $N, M$. Let $u$ denote the limit in $X_{p, 2}^{-\alpha, \alpha, T_{\omega}}$.

In the following, we give a brief discussion to show that the limit $u$ is a solution to (4). Clearly, $S(t) u_{0}^{N}$ and $\eta \Phi^{N}$ converge to $S(t) u_{0}$ and $\eta \Phi$ in $X_{p, 2}^{-\alpha, \alpha, T_{\omega}}$. It follows from (57) that $\mathcal{N}_{0}\left(u^{N}, u^{N}\right)$ converges to $\mathcal{N}_{0}(u, u)$ in $X_{p, 2}^{-\alpha, \alpha, T_{\omega}}$. In view of (72), (74), and (76), we see that $\mathcal{N}_{j}\left(u^{N}, u^{N}\right)$ is Cauchy in a slightly stronger space $X_{p, 2}^{-\alpha, 1-\alpha, T_{\omega}}, j=1,2$. Let $v_{j}$ denote the corresponding limit. Thus, from (66), we have

$$
u=S(t) u_{0}-\frac{1}{2} \mathcal{N}_{0}(u, u)-\frac{1}{2}\left(v_{1}+v_{2}\right)+\eta \Phi .
$$

Now, we need to show that $\mathcal{N}_{j}\left(u^{N}, u^{N}\right)$ indeed converges to $\mathcal{N}_{j}(u, u), j=1,2$. By symmetry, we only consider $\mathcal{N}_{1}(u, u)-\mathcal{N}_{1}\left(u^{N}, u^{N}\right)$. As before, we substitute (82) (and (66)) in the first factor $u$ (and $u^{N}$ ) of $\mathcal{N}_{1}(\cdot, \cdot)$, respectively. There are three contributions to consider.

(A) Contribution from the stochastic terms: We have

$$
\mathcal{N}_{1}(\eta \Phi, u)-\mathcal{N}_{1}\left(\eta \Phi^{N}, u^{N}\right)=\mathcal{N}_{1}\left(\eta\left(\Phi-\Phi^{N}\right), u\right)+\mathcal{N}_{1}\left(\eta \Phi^{N}, u-u^{N}\right) .
$$

From the estimate on (ii), we have

$$
\|(83)\|_{X_{p, 2}^{-\alpha, \alpha, T_{\omega}}} \lesssim \widetilde{L}_{\omega}^{N, \infty}\|u\|_{X_{p, 2}^{-\alpha, \alpha, T_{\omega}}}+L_{\omega}^{N}\left\|u^{N}-u\right\|_{X_{p, 2}^{-\alpha, \alpha, T_{\omega}}} \rightarrow 0
$$

as $N \rightarrow \infty$, since $\|u\|_{X_{p, 2}^{-a, \alpha}, T} \leq R_{\omega}$ and $\widetilde{L}_{\omega}^{N, \infty} \rightarrow 0$ uniformly on $\Omega_{\varepsilon}$.

(B) Contribution from $\mathcal{N}_{0}(\cdot, \cdot)$ : In this case, we consider

$$
\mathcal{N}_{1}\left(\mathcal{N}_{0}(u, u), u\right)-\mathcal{N}_{1}\left(\mathcal{N}_{0}\left(u^{N}, u^{N}\right), u^{N}\right) .
$$


Note that we have $\sigma_{1} \geq \sigma_{0}, \sigma_{2}, \sigma_{3}, \sigma_{4}$ from the definition of $\mathcal{N}_{1}(\cdot, \cdot)$ and $\mathcal{N}_{0}(\cdot, \cdot)$, see $(50)$ and (52). Indeed, we have $\sigma_{1} \geq \sigma_{0}, \sigma_{2}$ since we are on $A_{1}$ defined in (45), and also $\sigma_{1} \geq \sigma_{3}, \sigma_{4}$ since we are on the support of $\mathcal{N}_{0}(\cdot, \cdot)$ in the first factor of $\mathcal{N}_{1}(\cdot, \cdot)$. Once again, one can easily follow the argument in [Bourgain 1997, page 136] and show

$$
\|(84)\|_{X_{p, 2}^{-\alpha, \alpha, T_{\omega}}} \lesssim\left(\left\|u^{N}\right\|_{X_{p, 2}^{-\alpha, \alpha, T_{\omega}}}^{2}+\|u\|_{X_{p, 2}^{-\alpha, \alpha, T_{\omega}}}^{2}\left\|u^{N}-u\right\|_{X_{p, 2}^{-\alpha, \alpha, T_{\omega}}} \rightarrow 0 .\right.
$$

In treating $R_{\alpha}-R_{\alpha}^{N}$ defined in (58), one needs to proceed as before, using (77) and the estimate on (i).

(C) Contribution from $v_{j}$ and $\mathcal{N}_{j}\left(u^{N}, u^{N}\right), j=1$ or 2 : By symmetry, assume $j=1$. In this case, we have $\sigma_{1} \geq \sigma_{0}, \sigma_{2}$ but $\sigma_{3} \geq \sigma_{1}, \sigma_{4}$, thus we control (54) by the first term on the right-hand side [Bourgain 1997, (II.1) on page 126]. Now, we need to estimate

$$
\mathcal{N}_{1}\left(v_{1}, u\right)-\mathcal{N}_{1}\left(\mathcal{N}_{1}\left(u^{N}, u^{N}\right), u^{N}\right)=\mathcal{N}_{1}\left(v_{1}-\mathcal{N}_{1}\left(u^{N}, u^{N}\right), u\right)+\mathcal{N}_{1}\left(\mathcal{N}_{1}\left(u^{N}, u^{N}\right), u-u^{N}\right)=: \mathrm{I}+\mathrm{II} .
$$

Then, by proceeding as in [Bourgain 1997] with (56) and (72), we have

$$
\|\mathrm{II}\|_{X_{p, 2}^{-\alpha, 1-\alpha, T_{\omega}}} \lesssim I_{-\alpha, 1-\alpha}^{N}\left\|u-u^{N}\right\|_{X^{-(1-\alpha), \alpha, T_{\omega}}} \lesssim\left\|u-u^{N}\right\|_{X_{p, 2}^{-\alpha, \alpha, T_{\omega}}} \rightarrow 0 .
$$

By proceeding as in [Bourgain 1997, (II.1)] with $\left|n_{1}\right|^{\alpha}$ replaced by $\left|n_{1}\right|^{1-\alpha}$, followed by (56), we have

$$
\begin{aligned}
\|\mathrm{I}\|_{X_{p, 2}^{-\alpha, 1-\alpha, T_{\omega}}} & \lesssim\left\|v_{1}-\mathcal{N}_{1}\left(u^{N}, u^{N}\right)\right\|_{-(1-\alpha), 1-\alpha}\|u\|_{-(1-\alpha), \alpha} \\
& \lesssim\left\|v_{1}-\mathcal{N}_{1}\left(u^{N}, u^{N}\right)\right\|_{X_{p, 2}^{-\alpha, 1-\alpha, T_{\omega}}}\|u\|_{X_{p, 2}^{-\alpha, \alpha, T_{\omega}}} \rightarrow 0,
\end{aligned}
$$

since $v_{1}=\lim _{N \rightarrow \infty} \mathcal{N}_{1}\left(u^{N}, u^{N}\right)$ in $X_{p, 2}^{-\alpha, 1-\alpha, T_{\omega}}$ by definition.

Hence, we have $u=\Gamma_{u_{0}} u$ for each $\omega \in \Omega_{\varepsilon}$, so $u$ is a mild solution to (2) on [0, $\left.T_{\omega}\right]$. Let $\Omega^{(1)}=\Omega_{\varepsilon}$. Now, we can recursively construct

$$
\Omega^{(j+1)} \subset \Omega \backslash \bigcup_{k=1}^{j} \Omega^{(k)}
$$

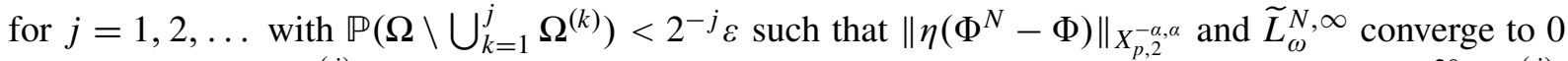
uniformly in each $\Omega^{(j)}$. Then, by repeating the argument, we can construct a solution $u$ on $\bigcup_{j=1}^{\infty} \Omega^{(j)}$. Note that $\mathbb{P}\left(\Omega \backslash \bigcup_{j=1}^{\infty} \Omega^{(j)}\right)=0$.

We have constructed a solution $u$ to (2) in $X_{p, 2}^{-\alpha, \alpha, T_{\omega}}$ with $u_{0} \in \hat{b}_{p, \infty}^{-\alpha^{\prime}}$. Since $u$ is a solution, the a priori estimate (73) holds with the regularity $(s, b)=\left(-\alpha^{\prime}, \alpha^{\prime}\right)$ in place of $(-\alpha, \alpha)$. Then, we easily see that $u \in X_{p, 2}^{-\alpha^{\prime}, \alpha^{\prime}, T_{\omega}}$ by redefining $R_{\omega}$ and $T_{\omega}$ with this regularity. In the remaining of the paper, we work only with the spatial regularity $s=-\alpha^{\prime}$, that is, there is no approximating sequences any more. Hence, for notational simplicity, we will use $-\alpha$ in place of $-\alpha^{\prime}$ to denote the spatial regularity of the solution in the following.

We still need to take care of several issues. Note that the temporal regularity $b=\alpha=\frac{1}{2}-\delta$ of the solution $u$ is less than $\frac{1}{2}$. In particular, we need to show that the solution $u$ is continuous from [0, $T_{\omega}$ ] into $\hat{b}_{p, \infty}^{-\alpha}$. We also need to show its uniqueness and continuous dependence on the initial data. 
From Proposition 4.5, $\eta \Phi \in C\left(\left[0, T_{\omega}\right] ; \hat{b}_{p, \infty}^{-\alpha}\right)$ a.s. Also, it follows from (67) with $b=\frac{1}{2}+\delta$, (69), (72), and symmetry on $\sigma_{1}$ and $\sigma_{2}$, that

$$
S(t) u_{0}+\mathcal{N}_{1}(u, u)+\mathcal{N}_{2}(u, u) \in X_{p, 2}^{-\alpha, \frac{1}{2}+\delta, T_{\omega}} \subset C\left(\left[0, T_{\omega}\right] ; \hat{b}_{p, \infty}^{-\alpha}\right)
$$

almost surely. Now, we consider $\mathcal{N}_{0}(u, u)$, that is, when $\sigma_{0}=$ MAX. Note that the contribution comes only from $M_{2}(u, u)$ defined in (51). Define

$$
\begin{aligned}
& \mathcal{N}_{3}(u, u)=\text { the contribution of } \mathcal{N}_{0}(u, u) \text { on }\left\{\max \left(\sigma_{1}, \sigma_{2}\right) \gtrsim\left\langle n n_{1} n_{2}\right\rangle^{1 / 100}\right\}, \\
& \mathcal{N}_{4}(u, u)=\mathcal{N}_{0}(u, u)-\mathcal{N}_{3}(u, u) .
\end{aligned}
$$

(a) First, we consider $\mathcal{N}_{3}(u, u)$, so $\max \left(\sigma_{1}, \sigma_{2}\right) \gtrsim\left\langle n n_{1} n_{2}\right\rangle^{1 / 100}$; say $\sigma_{1} \gtrsim\left\langle n n_{1} n_{2}\right\rangle^{1 / 100}$. Then, by (15) and Lemma 3.2, we have

$$
\left\|\mathcal{N}_{3}(u, u)\right\|_{X_{p, 2}^{-\alpha, \frac{1}{2}+\delta, T_{\omega}}} \lesssim\left\|\partial_{x}\left(u^{2}\right)\right\|_{X_{p, 2}^{-\alpha,-\frac{1}{2}+\delta, T_{\omega}}} \lesssim\left\|\partial_{x}\left(u^{2}\right)\right\|_{X^{-\alpha,-\frac{1}{2}+\delta, T_{\omega}}}
$$

By duality and (44), the right-hand side equals

$$
\begin{aligned}
& \sup _{\|d\|_{L_{n, \tau}^{2}=1}} \sum_{\substack{n, n_{1} \\
n=n_{1}+n_{2}}} \int_{\tau=\tau_{1}+\tau_{2}} \frac{\langle n\rangle^{1-\alpha} d(n, \tau)}{\sigma_{0}^{(1 / 2)-\delta}} \prod_{j=1}^{2} \frac{\left\langle n_{j}\right\rangle^{1-\alpha} c\left(n_{j}, \tau_{j}\right)}{\sigma_{j}^{\alpha}} d \tau d \tau_{1} \\
& \lesssim \sup _{\|d\|_{L_{n, \tau}^{2}=1}} \sum_{\substack{n, n_{1} \\
n=n_{1}+n_{2}}} \int_{\tau=\tau_{1}+\tau_{2}} d(n, \tau) \frac{c\left(n_{1}, \tau_{1}\right)}{\sigma_{1}^{\alpha-200 \delta}} \frac{c\left(n_{2}, \tau_{2}\right)}{\sigma_{2}^{\alpha}} d \tau d \tau_{1},
\end{aligned}
$$

where $c(n, \tau)$ is defined in (49). Thus, by the $L_{x, t}^{2}, L_{x, t}^{4}, L_{x, t}^{4}$-Hölder inequality along with Lemma 3.3 , (49), and (56), we conclude that

$$
\left\|\mathcal{N}_{3}(u, u)\right\|_{X_{p, 2}^{-\alpha, \frac{1}{2}+\delta, T_{\omega}}} \lesssim\|c\|_{L_{n, \tau}^{2}}^{2} \leq\|u\|_{X^{-(1-\alpha), \alpha}}^{2} \lesssim\|u\|_{X_{p, 2}^{-\alpha, \alpha}}^{2}<\infty
$$

(b) Now, consider $\mathcal{N}_{4}(u, u)$, so $\max \left(\sigma_{1}, \sigma_{2}\right) \ll\left\langle n n_{1} n_{2}\right\rangle^{1 / 100}$. It suffices to show that $\mathcal{N}_{0}(u, u) \in X_{p, 1}^{-\alpha, 0, T_{\omega}}$, since $X_{p, 1}^{-\alpha, 0, T_{\omega}} \subset C\left(\left[0, T_{\omega}\right] ; \hat{b}_{p, \infty}^{-\alpha}\right)$. Then, by Cauchy-Schwarz inequality, Lemma 5.3 and duality, we have

$$
\begin{aligned}
\left\|\mathcal{N}_{4}(u, u)\right\|_{X_{p, 1}^{-\alpha, 0, T_{\omega}}} & \leq\left\|\partial_{x}\left(u^{2}\right)\right\|_{X_{2,1}^{-\alpha,-1, T_{\omega}}} \leq\|\|\langle n\rangle^{-\alpha}\left\langle\tau-n^{3}\right\rangle^{-1} \chi_{\Omega(n)}\left(\tau-n^{3}\right) \widehat{\partial_{x}\left(u^{2}\right)}(n, \tau)\left\|_{L_{\tau}^{1}}\right\|_{L_{n}^{2}} \\
& \leq\left\|\left\langle\tau-n^{3}\right\rangle^{-\frac{1}{2}+\delta} \chi_{\Omega(n)}\left(\tau-n^{3}\right)\right\|_{L_{\tau}^{2}}\left\|\partial_{x}\left(u^{2}\right)\right\|_{-\alpha,-\frac{1}{2}-\delta} \\
& \lesssim \sup _{\|d\|_{L_{n, \tau}^{2}}=1} \sum_{n, n_{1}} \int_{n=n_{1}+n_{2}} \frac{\langle n\rangle^{1-\alpha} d(n, \tau)}{\sigma_{0}^{(1 / 2)+\delta}} \prod_{j=1}^{2} \frac{\left\langle n_{j}\right\rangle^{1-\alpha} c\left(n_{j}, \tau_{j}\right)}{\sigma_{j}^{\alpha}} d \tau d \tau_{1} \\
& \lesssim \sup _{\|d\|_{L_{n, \tau}^{2}}=1} \sum_{n=n_{1}} \int_{n=n_{1}+n_{2}} d(n, \tau) \frac{c\left(n_{1}, \tau_{1}\right)}{\sigma_{1}^{\alpha}} \frac{c\left(n_{2}, \tau_{2}\right)}{\sigma_{2}^{\alpha}} d \tau d \tau_{1} .
\end{aligned}
$$

The rest follows as before. Hence, the solution $u$ is continuous from $\left[0, T_{\omega}\right]$ to $\hat{b}_{p, \infty}^{-\alpha}$. 
Lastly, we show the uniqueness and the continuous dependence of the solutions on the initial data. Let $u$ and $v$ be the mild solutions of (2) on $\left[0, T_{\omega}\right]$ with initial data $u_{0}$ and $v_{0}$; then

$$
u-v=\Gamma_{u_{0}} u-\Gamma_{v_{0}} v=S(t)\left(u_{0}-v_{0}\right)-\frac{1}{2}(\mathcal{N}(u, u)-\mathcal{N}(v, v)),
$$

where $\Gamma$ is defined in (66). Moreover, assume that

$$
\left\|u_{0}\right\|_{\hat{b}_{p, \infty}^{-\alpha}} \leq R, \quad\left\|v_{0}\right\|_{\hat{b}_{p, \infty}^{-\alpha}} \leq R, \quad\|u\|_{X_{p, 2}^{-\alpha, \alpha, T_{\omega}}} \leq R, \quad\|v\|_{X_{p, 2}^{-\alpha, \alpha, T_{\omega}}} \leq R .
$$

Let $\widetilde{\mathcal{N}}_{j}(u, v):=-\frac{1}{2}\left(\mathcal{N}_{j}(u, u)-\mathcal{N}_{j}(v, v)\right)$ for $j=1, \ldots, 4$. First, note that $\left\|\widetilde{\mathcal{N}}_{4}(u, v)\right\|_{X_{p, 1}^{-\alpha, \varepsilon} T_{\omega}} \lesssim R^{2}<\infty$ from (a slight variation of) Case (b), and we have

$$
\left\|(u-v)-\widetilde{\mathcal{N}}_{4}(u, v)\right\|_{X_{p, 1}^{-\alpha, T_{\omega}}} \leq\left\|S(t)\left(u_{0}-v_{0}\right)+\sum_{j=1}^{3} \widetilde{\mathcal{N}}_{j}(u, v)\right\|_{X_{p, 2}^{-\alpha, \frac{1}{2}+\delta, T_{\omega}}} \lesssim C_{1}(R)<\infty
$$

by Cauchy-Schwarz inequality with $\varepsilon<\delta$, followed by (67), (69), (72), Case (a), and (87). Then, by interpolation and Cauchy-Schwarz inequality, we have

$$
\|u-v\|_{C\left(\left[0, T_{\omega}\right] ; \hat{b}_{p, \infty}^{-\alpha}\right)} \lesssim\|u-v\|_{X_{p, 1}^{-\alpha, 0, T_{\omega}}} \lesssim\|u-v\|_{X_{p, 1}^{-\alpha,-\delta-, T_{\omega}}}^{\beta}\|u-v\|_{X_{p, 1}^{-\alpha, \varepsilon, T_{\omega}}}^{1-\beta} \lesssim C_{2}(R)\|u-v\|_{X_{p, 2}^{-\alpha, \frac{1}{2}-\delta, T_{\omega}}}^{\beta}
$$

with $\beta=\frac{\varepsilon}{\varepsilon+\delta+} \in(0,1)$. From (67) and the nonlinear estimates (see (68), (72), (74), (76)), we have

$$
\|u-v\|_{X_{p, 2}^{-\alpha, \frac{1}{2}-\delta, T_{\omega}}} \lesssim\left\|u_{0}-v_{0}\right\|_{\hat{b}_{p, \infty}^{-\alpha}}+C_{3}(R) T_{\omega}^{\theta}\|u-v\|_{X_{p, 2}^{-\alpha, \frac{1}{2}-\delta, T_{\omega}}} .
$$

Hence, for sufficiently small $T>0$, we have

$$
\|u-v\|_{X_{p, 2}^{-\alpha, \frac{1}{2}-\delta, T_{\omega}}} \lesssim\left\|u_{0}-v_{0}\right\|_{\hat{b}_{p, \infty}^{-\alpha}} .
$$

Therefore, it follows from (88) and (89) that the solution map is Hölder continuous with the bound

$$
\|u-v\|_{C\left(\left[0, T_{\omega}\right] ; \hat{b}_{p, \infty}^{-\alpha}\right)} \leq C_{4}(R)\left\|u_{0}-v_{0}\right\|_{\hat{b}_{p, \infty}^{-\alpha}}^{\beta} .
$$

In particular, the solution is unique. This completes the proof of Theorem 1.

\section{Acknowledgments}

The author thanks Prof. Jeremy Quastel and Prof. Catherine Sulem for suggesting this problem.

\section{References}

[Bourgain 1993] J. Bourgain, "Fourier transform restriction phenomena for certain lattice subsets and applications to nonlinear evolution equations, II: The KdV-equation”, Geom. Funct. Anal. 3:3 (1993), 209-262. MR 95d:35160b Zbl 0787.35098

[Bourgain 1997] J. Bourgain, "Periodic Korteweg-de Vries equation with measures as initial data", Selecta Math. (N.S.) 3:2 (1997), 115-159. MR 2000i:35173 Zbl 0891.35138

[Bourgain 1999] J. Bourgain, "Nonlinear Schrödinger equations", pp. 3-157 in Hyperbolic equations and frequency interactions (Park City, UT, 1995), edited by L. Caffarelli and E. Weinan, IAS/Park City Math. Ser. 5, Amer. Math. Soc., Providence, RI, 1999. MR 2000c:35216 Zbl 0952.35127 
[Christ et al. 2003] M. Christ, J. Colliander, and T. Tao, "Asymptotics, frequency modulation, and low regularity ill-posedness for canonical defocusing equations", Amer. J. Math. 125:6 (2003), 1235-1293. MR 2005d:35223 Zbl 1048.35101

[Colliander and Oh 2009] J. Colliander and T. Oh, "Almost sure well-posedness of the cubic nonlinear Schrödinger equation below $L^{2}(\mathbb{T})$ ", preprint, 2009.

[Colliander et al. 2003] J. Colliander, M. Keel, G. Staffilani, H. Takaoka, and T. Tao, "Sharp global well-posedness for KdV and modified KdV on $\mathbb{R}$ and T", J. Amer. Math. Soc. 16:3 (2003), 705-749. MR 2004c:35352 Zbl 1025.35025

[Da Prato 2004] G. Da Prato, Kolmogorov equations for stochastic PDEs, Birkhäuser Verlag, Basel, 2004. MR 2005m:60002 Zbl 1066.60061

[De Bouard et al. 2004] A. De Bouard, A. Debussche, and Y. Tsutsumi, "Periodic solutions of the Korteweg-de Vries equation driven by white noise", SIAM J. Math. Anal. 36:3 (2004), 815-855. MR 2005k:60194 Zbl 1075.35121

[Kappeler and Topalov 2006] T. Kappeler and P. Topalov, "Global wellposedness of KdV in $H^{-1}(\mathbb{T}, \mathbb{R})$ ", Duke Math. J. 135:2 (2006), 327-360. MR 2007i:35199 Zbl 1106.35081

[Kenig et al. 1993] C. E. Kenig, G. Ponce, and L. Vega, "The Cauchy problem for the Korteweg-de Vries equation in Sobolev spaces of negative indices”, Duke Math. J. 71:1 (1993), 1-21. MR 94g:35196 Zbl 0787.35090

[Kenig et al. 1996] C. E. Kenig, G. Ponce, and L. Vega, "A bilinear estimate with applications to the KdV equation", J. Amer. Math. Soc. 9:2 (1996), 573-603. MR 96k:35159 Zbl 0848.35114

[Kenig et al. 2001] C. E. Kenig, G. Ponce, and L. Vega, "On the ill-posedness of some canonical dispersive equations", Duke Math. J. 106:3 (2001), 617-633. MR 2002c:35265 Zbl 1034.35145

[Oh 2009a] T. Oh, "Invariance of the white noise for KdV", Comm. Math. Phys. 292:1 (2009), 217-236.

[Oh 2009b] T. Oh, "White noise for KdV and mKdV on the circle", preprint, 2009. To appear in RIMS Kôkyûroku Bessatsu.

[Roynette 1993] B. Roynette, "Mouvement brownien et espaces de Besov", Stochastics Stochastics Rep. 43:3-4 (1993), 221260. MR 95h:60042 Zbl 0808.60071

Received 2 Jan 2009. Revised 28 Aug 2009. Accepted 19 Oct 2009.

TADAHIRO OH: oh@math.toronto.edu

Department of Mathematics, University of Toronto, 40 St. George Street, Rm 6290, Toronto, ON M5S 2E4, Canada 


\title{
Analysis \& PDE
}

pjm.math.berkeley.edu/apde

EDITORS

\author{
EDITOR-IN-CHIEF \\ Maciej Zworski \\ University of California \\ Berkeley, USA
}

BOARD OF EDITORS

\begin{tabular}{|c|c|c|c|}
\hline Michael Aizenman & $\begin{array}{l}\text { Princeton University, USA } \\
\text { aizenman@math.princeton.edu }\end{array}$ & Nicolas Burq & $\begin{array}{l}\text { Université Paris-Sud 11, France } \\
\text { nicolas.burq @math.u-psud.fr }\end{array}$ \\
\hline Luis A. Caffarelli & $\begin{array}{l}\text { University of Texas, USA } \\
\text { caffarel@math.utexas.edu }\end{array}$ & un-Yung Alice Chang & $\begin{array}{l}\text { Princeton University, USA } \\
\text { chang@math.princeton.edu }\end{array}$ \\
\hline Michael Christ & $\begin{array}{l}\text { University of California, Berkeley, USA } \\
\text { mchrist@math.berkeley.edu }\end{array}$ & Charles Fefferman & $\begin{array}{l}\text { Princeton University, USA } \\
\text { cf@math.princeton.edu }\end{array}$ \\
\hline Ursula Hamenstaedt & $\begin{array}{l}\text { Universität Bonn, Germany } \\
\text { ursula@math.uni-bonn.de }\end{array}$ & Nigel Higson & $\begin{array}{l}\text { Pennsylvania State Univesity, USA } \\
\text { higson@ math.psu.edu }\end{array}$ \\
\hline Vaughan Jones & $\begin{array}{l}\text { University of California, Berkeley, USA } \\
\text { vfr@math.berkeley.edu }\end{array}$ & Herbert Koch & $\begin{array}{l}\text { Universität Bonn, Germany } \\
\text { koch@math.uni-bonn.de }\end{array}$ \\
\hline Izabella Laba & $\begin{array}{l}\text { University of British Columbia, Canada } \\
\text { ilaba@math.ubc.ca }\end{array}$ & Gilles Lebeau & $\begin{array}{l}\text { Université de Nice Sophia Antipolis, France } \\
\text { lebeau@unice.fr }\end{array}$ \\
\hline László Lempert & $\begin{array}{l}\text { Purdue University, USA } \\
\text { lempert@math.purdue.edu }\end{array}$ & Richard B. Melrose & $\begin{array}{l}\text { Massachussets Institute of Technology, USA } \\
\text { rbm@math.mit.edu }\end{array}$ \\
\hline Frank Merle & $\begin{array}{l}\text { Université de Cergy-Pontoise, France } \\
\text { Frank.Merle@u-cergy.fr }\end{array}$ & William Minicozzi II & $\begin{array}{l}\text { Johns Hopkins University, USA } \\
\text { minicozz@ math.jhu.edu }\end{array}$ \\
\hline Werner Müller & $\begin{array}{l}\text { Universität Bonn, Germany } \\
\text { mueller@math.uni-bonn.de }\end{array}$ & Yuval Peres & $\begin{array}{l}\text { University of California, Berkeley, USA } \\
\text { peres@stat.berkeley.edu }\end{array}$ \\
\hline Gilles Pisier & $\begin{array}{l}\text { Texas A\&M University, and Paris } 6 \\
\text { pisier@math.tamu.edu }\end{array}$ & Tristan Rivière & $\begin{array}{l}\text { ETH, Switzerland } \\
\text { riviere@math.ethz.ch }\end{array}$ \\
\hline Igor Rodnianski & $\begin{array}{l}\text { Princeton University, USA } \\
\text { irod@ math.princeton.edu }\end{array}$ & Wilhelm Schlag & $\begin{array}{l}\text { University of Chicago, USA } \\
\text { schlag@math.uchicago.edu }\end{array}$ \\
\hline Sylvia Serfaty & $\begin{array}{l}\text { New York University, USA } \\
\text { serfaty@ cims.nyu.edu }\end{array}$ & Yum-Tong Siu & $\begin{array}{l}\text { Harvard University, USA } \\
\text { siu@math.harvard.edu }\end{array}$ \\
\hline Terence Tao & $\begin{array}{l}\text { University of California, Los Angeles, USA } \\
\text { tao@math.ucla.edu }\end{array}$ & A Michael E. Taylor & $\begin{array}{l}\text { Univ. of North Carolina, Chapel Hill, USA } \\
\text { met@math.unc.edu }\end{array}$ \\
\hline Gunther Uhlmann & $\begin{array}{l}\text { University of Washington, USA } \\
\text { gunther@math.washington.edu }\end{array}$ & András Vasy & $\begin{array}{l}\text { Stanford University, USA } \\
\text { andras@math.stanford.edu }\end{array}$ \\
\hline Virgil Voiculescu & $\begin{array}{l}\text { University of California, Berkeley, USA } \\
\text { dvv@math.berkeley.edu }\end{array}$ & Steven Zelditch & $\begin{array}{l}\text { Johns Hopkins University, USA } \\
\text { szelditch@math.jhu.edu }\end{array}$ \\
\hline
\end{tabular}

\section{PRODUCTION}

apde@mathscipub.org

Paulo Ney de Souza, Production Manager Sheila Newbery, Production Edito

Silvio Levy, Senior Production Editor

See inside back cover or pjm.math.berkeley.edu/apde for submission instructions.

The subscription price for 2009 is US \$120/year for the electronic version, and \$180/year for print and electronic. Subscriptions, requests for back issues from the last three years and changes of subscribers address should be sent to Mathematical Sciences Publishers, Department of Mathematics, University of California, Berkeley, CA 94720-3840, USA.

Analysis \& PDE, at Mathematical Sciences Publishers, Department of Mathematics, University of California, Berkeley, CA 94720-3840 is published continuously online. Periodical rate postage paid at Berkeley, CA 94704, and additional mailing offices.

APDE peer-review and production is managed by EditFLOW ${ }^{\mathrm{TM}}$ from Mathematical Sciences Publishers.

PUBLISHED BY

mathematical sciences publishers

http://www.mathscipub.org

A NON-PROFIT CORPORATION

Typeset in IATEX

Copyright (C2009 by Mathematical Sciences Publishers 


\section{ANALYSIS \& PDE}

\section{Volume $2 \quad$ No. $3 \quad 2009$}

Global existence of smooth solutions of a 3D log-log energy-supercritical wave equation

TRISTAN ROY

Periodic stochastic Korteweg-de Vries equation with additive space-time white noise

TADAHIRO OH

Stability for strongly coupled critical elliptic systems in a fully inhomogeneous medium

305

OLIVIER DRUET and EMMANUEL HEBEY

Global regularity for a logarithmically supercritical hyperdissipative Navier-Stokes equation 361

TERENCE TAO 